\title{
SPECTRAL METHODS IN POLAR COORDINATES FOR THE STOKES PROBLEM. APPLICATION TO COMPUTATION IN UNBOUNDED DOMAINS
}

\author{
LAURENCE HALPERN
}

\begin{abstract}
We present spectral methods for solving the Stokes problem in a circular domain. Their main feature is the uniform inf-sup condition, which allows for optimal error estimates. We apply them to the resolution of exterior problems by coupling with the transparent boundary condition.
\end{abstract}

\section{INTRODUCTION}

When solving a problem in an unbounded domain, it is customary to introduce an artificial boundary, and to prescribe on it a so-called "transparent boundary condition", which replaces the missing part of the domain. This leads to a wellposed problem in a bounded domain, with an integral boundary condition. In [10] a method has been introduced for coupling finite elements and the integral equation for the Laplace equation in an exterior domain. This method has been extended to the Stokes problem in [15] and to the Maxwell equations in [13]. Other numerical methods have been developed, coupling finite elements in the interior and spectral decompositions on the boundary (see for instance $[11,12]$ ).

The finite element method is often preferable when dealing with complicated geometries. Nevertheless, in two dimensions, if the artificial boundary is chosen to be a circle, the transparent boundary condition has a very simple expression in the angular coordinate $\theta$. It seems most natural to take advantage of it to approximate the solution with polynomials in $r$ and trigonometric polynomials in $\theta$. Successful computations using spectral methods have been presented in [4]. A theoretical formalism is the aim of the present paper.

As an interesting illustration we chose the steady Stokes problem in two dimensions. We first consider the problem with homogeneous Dirichlet boundary condition in the disc of center 0 and radius 1 . The weak formulation reads (the notations can be found in $\S 2$ ):

$$
\begin{aligned}
& \text { find }(\mathbf{u}, p) \text { in } \mathbf{H}_{0}^{1}(\Omega) \times L_{0}^{2}(\Omega) \text { such that: } \\
& \left\{\begin{array}{l}
\forall \mathbf{v} \in \mathbf{H}_{0}^{1}(\Omega), \quad a(\mathbf{u}, \mathbf{v})+b(\mathbf{v}, p)=\langle\mathbf{f}, \mathbf{v}\rangle, \\
\forall q \in L_{0}^{2}(\Omega), \quad b(\mathbf{u}, q)=0
\end{array}\right.
\end{aligned}
$$

Received by the editor March 14, 1994 and, in revised form, December 15, 1994.

1991 Mathematics Subject Classification. Primary 35C10, 35G15, 65M70, 65T10. 
Owing to a well-known result of Babuška and Brezzi (see [8]), the most delicate property to fulfill for well-posedness is the so-called inf-sup condition: there exists a positive number $C>0$ such that

$$
\inf _{q \in M} \sup _{\mathbf{v} \in X} \frac{b(\mathbf{v}, q)}{\|\mathbf{v}\|_{X}\|q\|_{M}} \geq C
$$

We recall in $\S 2$ that this condition is fulfilled for this problem in any regular geometry. In our case, the best value $C$ can be given explicitly: $1 / \sqrt{2}$ (Theorem 2.1 ). In most cases we find in the literature (rectangular domains) that the use of spectral methods produces "parasitic modes", which perturb the computation of the pressure (see for instance $[2,17]$ ). This is expressed by the fact that the constant $C$ in the discrete inf-sup condition tends to 0 as the number of modes increases. The spectral methods we suggest here lead to uniform inf-sup conditions.

We start with the Galerkin method ( $\S 3)$. Here, $N$ and $K$ are two integers greater than or equal to $2, \mathbf{S}_{K}([0,2 \pi])$ is the space of trigonometric polynomials in $\theta$ of degree less than or equal to $K$, and $\mathbf{P}_{N}([0,1])$ the space of polynomials in $r$ of degree less than or equal to $N$. The approximation is made in $X_{N} \times M_{N}$, where $M_{N}=L_{0}^{2}(\Omega) \cap\left(\mathbf{S}_{N-1} \otimes \mathbf{P}_{N-1}\right)$ and $X_{N}=\mathbf{H}_{0}^{1}(\Omega) \cap\{\mathbf{u}$ s.t. div $\mathbf{u}$ and curl $\mathbf{u}$ belongs to $\left.\mathbf{S}_{N-1} \otimes \mathbf{P}_{N-1}\right\}$. The approximate problem is:

$$
\begin{aligned}
& \text { find }\left(\mathbf{u}_{N}, p_{N}\right) \text { in } X_{N} \times M_{N} \text { such that: } \\
& \begin{cases}\forall \mathbf{v} \in X_{N}, & a\left(\mathbf{u}_{N}, \mathbf{v}\right)+b\left(\mathbf{v}, p_{N}\right)=\langle\mathbf{f}, \mathbf{v}\rangle, \\
\forall q \in M_{N}, & b\left(\mathbf{u}_{h}, q\right)=0 .\end{cases}
\end{aligned}
$$

A convenient decomposition of vector fields on the circle gives the inf-sup condition, and the constant is still equal to $1 / \sqrt{2}$. We then give two projection theorems in the weighted Sobolev spaces on $(0,1)$,

$$
H_{r}^{p}(0,1)=\left\{\psi \in \mathcal{D}^{\prime}(0,1), \int_{0}^{1} r\left|\psi^{(j)}\right|^{2}(r) d r<+\infty \text { for } 0 \leq j \leq p\right\} .
$$

The first one, in $L_{r}^{2}$, is classical. The second one, in $H_{r}^{1}$, is more delicate. The technique of the proof is inspired by [1], but the lack of a Hardy inequality requires new partial results. These theorems lead to "optimal" error estimates: if $\mathbf{f}$ belongs to $H^{p}$ for $p \geq 0$, then $\left\|\mathbf{u}-\mathbf{u}_{N}\right\|_{X}+\left\|p-p_{N}\right\|_{M} \leq C N^{-1-p}\|\mathbf{f}\|_{p}$.

In $\S 4$, we present a pseudospectral method. It relies on a Gauss-Lobatto quadrature formula on $[0,1]$ for the weight $r$. The constant in the inf-sup condition remains the same. For the error estimates we need results on polynomial interpolation in $H_{r}^{p}(0,1)$. Again, we use the strategy in [1], but some new lemmas are necessary. The error estimates are still "optimal".

With these tools, we are now able to study the problem in an unbounded domain $(\S 5)$. We first reduce it to a disc by giving the transparent operator, and writing the variational formulation. For the discrete formulation, we introduce the Galerkin method. In both cases, continuous and discrete, the constant in the inf-sup condition is equal to 1 , which in turn allows for optimal error estimates.

\section{The Stokes PROBlem IN A DISC}

2.1. Variational formulation in a bounded domain. Let $\Omega$ be a bounded open connected subset of $\mathbf{R}^{2}$, with smooth boundary $\Gamma$. The Stokes problem in $\Omega$ with 
homogeneous Dirichlet boundary data reads: find $(\mathbf{u}, p)$ such that

$$
\left\{\begin{array}{l}
-\Delta \mathbf{u}+\nabla p=\mathbf{f} \quad \text { in } \Omega \\
\nabla \cdot \mathbf{u}=0 \text { in } \Omega \\
\mathbf{u}=\mathbf{0} \text { on } \Gamma .
\end{array}\right.
$$

Here, $\nabla, \nabla \cdot$, and $\Delta$ denote respectively the gradient, divergence, and Laplace operators: $\nabla p=\left(\frac{\partial p}{\partial x_{1}}, \frac{\partial p}{\partial x_{2}}\right), \nabla \cdot \mathbf{u}=\frac{\partial u_{1}}{\partial x_{1}}+\frac{\partial u_{2}}{\partial x_{2}}, \Delta u=\frac{\partial^{2} u}{\partial x_{1}^{2}}+\frac{\partial^{2} u}{\partial x_{2}^{2}}$. For any positive integer $m$, we denote by $H^{m}(\Omega)$ the Sobolev space of distributions in $\Omega$ whose derivatives up to order $m$ belong to $L^{2}(\Omega)$, furnished with the inner product

$$
(v, w)_{0}=\int_{\Omega} v(x) \bar{w}(x) d x, \quad(v, w)_{m}=\sum_{|k| \leq m}\left(D^{k} v, D^{k} w\right)_{0} .
$$

The corresponding norm is denoted by $\|v\|_{m}\left(H^{0}(\Omega)=L^{2}(\Omega)\right.$ ). By $\mathbf{H}^{m}(\Omega)$ (resp. $\left.\mathbf{L}^{2}(\Omega)\right)$ we denote the space of vector-valued distributions whose two components belong to $H^{m}(\Omega)$ (resp. $L^{2}(\Omega)$ ), whereas $\|\cdot\|_{m}$ and $(\cdot, \cdot)_{m}$ are the norm and scalar product either in $H^{m}(\Omega)$ or in $\mathbf{H}^{m}(\Omega)$. Furthermore, $L_{0}^{2}(\Omega)$ is the space of distributions in $L^{2}(\Omega)$ such that $(v, 1)_{0}=0, H_{0}^{1}(\Omega)$ the closure of $\mathcal{D}(\Omega)$ in $H^{1}(\Omega)$, or equivalently, $H_{0}^{1}(\Omega)=\left\{v \in H^{1}(\Omega), v=0\right.$ on $\left.\Gamma\right\}$, and $H^{-1}(\Omega)$ is the dual space of $H_{0}^{1}(\Omega)$. The duality between $H^{-1}(\Omega)$ and $H_{0}^{1}(\Omega)$ will be denoted by $\langle\cdot, \cdot\rangle$. In view of the Poincaré-Friedrichs inequality, the seminorm defined by $|v|_{1}=\|\nabla v\|_{0}$ is a norm on $H_{0}^{1}(\Omega)$, equivalent to the $\|\cdot\|_{1}$ norm. Finally, $H^{1 / 2}(\Gamma)$ is the space of traces on $\Gamma$ of the elements of $H^{1}(\Omega)$, and $H^{-1 / 2}(\Gamma)$ its dual space. The duality between $H^{1 / 2}(\Gamma)$ and $H^{-1 / 2}(\Gamma)$ will be denoted by $\langle\cdot, \cdot\rangle_{\Gamma}$.

According to the following result (cf. [8]), problem (2.1) is well-posed.

Theorem A. If $\mathbf{f}$ belongs to $\mathbf{H}^{-1}(\Omega)$, there exists a unique solution $(\mathbf{u}, p)$ to $(2.1)$ in $\mathbf{H}^{1}(\Omega) \times L_{0}^{2}(\Omega)$ and

$$
\|\mathbf{u}\|_{1}+\|p\|_{0} \leq C\|\mathbf{f}\|_{-1} .
$$

Moreover, if $\mathbf{f}$ belongs to $\mathbf{H}^{m}(\Omega)$, then $(\mathbf{u}, p)$ belongs to $\mathbf{H}^{m+2}(\Omega) \times H^{m+1}(\Omega)$ and

$$
\|\mathbf{u}\|_{m+2}+\|p\|_{m+1} \leq C\|\mathbf{f}\|_{m}
$$

The existence and uniqueness rely on the following weak formulation: let $X$ be the Hilbert space $\mathbf{H}_{0}^{1}(\Omega)$ provided with the $|\cdot|_{1}$ inner product and $M$ be $L_{0}^{2}(\Omega)$ provided with the $L^{2}$ scalar product:

$$
\begin{gathered}
(\mathbf{u}, \mathbf{v})_{X}=(\nabla \mathbf{u}, \nabla \mathbf{v})_{0}=\left(\nabla u_{1}, \nabla v_{1}\right)_{0}+\left(\nabla u_{2}, \nabla v_{2}\right)_{0} ; \quad\|\mathbf{u}\|_{X}=\|\nabla \mathbf{u}\|_{0} ; \\
(u, v)_{M}=(u, v)_{0} ; \quad\|u\|_{M}=\|u\|_{0} .
\end{gathered}
$$

We define the bilinear forms $a$ and $b$, and the linear form $L$, by

$$
\left\{\begin{array}{l}
a(\mathbf{u}, \mathbf{v})=(\nabla \mathbf{u}, \nabla \mathbf{v})_{0}=(\mathbf{u}, \mathbf{v})_{X}, \\
b(\mathbf{v}, q)=-(q, \nabla \cdot \mathbf{v})_{0}, \\
L(\mathbf{v})=\langle\mathbf{f}, \mathbf{v}\rangle .
\end{array}\right.
$$

The weak formulation reads: find $(\mathbf{u}, p)$ in $X \times M$ such that

$$
\left\{\begin{array}{l}
\forall \mathbf{v} \in X, a(\mathbf{u}, \mathbf{v})+b(\mathbf{v}, p)=L(\mathbf{v}), \\
\forall q \in M, b(\mathbf{u}, q)=0
\end{array}\right.
$$

We introduce the subspace $V=\{\mathbf{v} \in X, \nabla \cdot \mathbf{v}=0\}$. Theorem A is a consequence of the following general result (cf. [8]): 
Theorem B. Suppose the following assumptions are fulfilled:

(i) $a$ is bilinear continuous on $X$ and there exists a positive constant $\alpha$ such that for any $v$ in $V, a(v, v) \geq \alpha\|v\|_{X}^{2}$,

(ii) $b$ is bilinear continuous on $X \times M$,

(iii) the inf-sup condition of Babuška-Brezzi is satisfied: there exists a real number $C>0$ such that

$$
\inf _{q \in M} \sup _{\mathbf{v} \in X} \frac{b(\mathbf{v}, q)}{\|\mathbf{v}\|_{X}\|q\|_{M}} \geq C
$$

(iv) $L$ is linear continuous on $X$.

Then problem (2.3) has a unique solution.

We shall from now on consider the case where $\Omega=D(0,1)$ is the unitary disc with center 0 and radius 1 .

2.2. The inf-sup condition in $D(0,1)$. In order to construct a "good" approximation, we shall first calculate the constant $C$.

Theorem 2.1. For $\Omega=D(0,1)$, one has

$$
\inf _{q \in M} \sup _{\mathbf{v} \in X} \frac{b(\mathbf{v}, q)}{\|\mathbf{v}\|_{X}\|q\|_{M}}=\frac{1}{\sqrt{2}} .
$$

Proof. Following [8] or [17], we write

$$
\inf _{q \in M} \sup _{\mathbf{v} \in X} \frac{b(\mathbf{v}, q)}{\|\mathbf{v}\|_{X}\|q\|_{M}}=\inf _{q \in M} \frac{\|\mathbf{w}(q)\|_{X}}{\|q\|_{M}},
$$

where $\mathbf{w}(q)$ is the unique solution to the problem

$$
\mathbf{w} \in X, \forall \mathbf{v} \in X, \quad a(\mathbf{w}, \mathbf{v})=b(\mathbf{v}, q),
$$

which can be rewritten as

$$
\left\{\begin{array}{l}
\mathbf{w} \in H_{0}^{1}(\Omega) \\
\Delta \mathbf{w}+\nabla q=\mathbf{0}
\end{array}\right.
$$

We shall write $\mathbf{w}$ as a function of $q$. This can easily be done in polar coordinates. A basis in $L^{2}(\Gamma)$ is given by the sequence $H_{m}(\theta)=\frac{1}{\sqrt{2 \pi}} \exp (\operatorname{im} \theta)$ for $m \in \mathbf{Z}$. A basis in $\mathbf{L}^{2}(\Gamma)$ is given by the two sequences $\mathbf{V}_{m}(\theta)$ and $\mathbf{W}_{m}(\theta)$ for $m \in \mathbf{Z}$, with

$$
\left\{\begin{array}{l}
2 \mathbf{V}_{m}(\theta)=H_{m}(\theta)\left(\mathbf{e}_{1}+i \mathbf{e}_{2}\right)=H_{m+1}(\theta)\left(\mathbf{e}_{r}+i \mathbf{e}_{\theta}\right), \\
2 \mathbf{W}_{m}(\theta)=H_{m}(\theta)\left(\mathbf{e}_{1}-i \mathbf{e}_{2}\right)=H_{m-1}(\theta)\left(\mathbf{e}_{r}-i \mathbf{e}_{\theta}\right) .
\end{array}\right.
$$

Here, $\left\{\mathbf{e}_{1}, \mathbf{e}_{2}\right\}$ is the usual basis in $\mathbf{R}^{2},\left\{\mathbf{e}_{r}, \mathbf{e}_{\theta}\right\}$ the moving basis. Note that $\mathbf{V}_{m}(\theta)=\overline{\mathbf{W}_{-m}(\theta)}$. The sequence $H_{m}$ is orthonormal in $L^{2}(\Gamma)$ (the norm is 1 ), the sequence $\left\{\mathbf{V}_{m}, \mathbf{W}_{m}\right\}$ is orthonormal in $\mathbf{L}^{2}(\Gamma)$ (the norm is $\left.\frac{1}{\sqrt{2}}\right)$.

Let us write $q$ and $\mathbf{w}$ in separate variables:

$$
\begin{gathered}
q(r, \theta)=\sum_{m \in \mathbf{Z}} q_{m}(r) H_{m}(\theta) \\
\mathbf{w}(r, \theta)=\sum_{m \in \mathbf{Z}} v_{m}(r) \mathbf{V}_{m}(\theta)+\sum_{m \in \mathbf{Z}} w_{m}(r) \mathbf{W}_{m}(\theta) .
\end{gathered}
$$

Since $\mathbf{w}$ and $q$ are real functions, we have, for any $m, q_{m}=\bar{q}_{-m}$ and $v_{m}=\bar{w}_{-m}$. 
SPECTRAL METHODS IN POLAR COORDINATES FOR THE STOKES PROBLEM 511

We introduce $L_{r}^{2}(0,1)=\left\{\psi \in \mathcal{D}^{\prime}(0,1), \int_{0}^{1} r|\psi|^{2}(r) d r<+\infty\right\}$, furnished with the natural norm $\|\psi\|_{L_{r}^{2}}^{2}=\int_{0}^{1} r|\psi|^{2}(r) d r$, and the corresponding inner product $(\cdot, \cdot)_{r}$. The norms of $q$ in $L^{2}(\Omega)$ and of $\mathbf{w}$ in $X$ are given by

$$
\|q\|_{0}^{2}=\sum_{m \in \mathbf{Z}}\left\|q_{m}\right\|_{L_{r}^{2}}^{2} \quad \text { and } \quad\|\mathbf{w}\|_{X}^{2}=\sum_{m \in \mathbf{Z}}\left(\left\|\frac{d v_{m}}{d r}\right\|_{L_{r}^{2}}^{2}+\left\|\frac{m}{r} v_{m}\right\|_{L_{r}^{2}}^{2}\right) .
$$

According to [14], for any $m \neq 0$, we have $v_{m}(0)=0$. Define, for any $m$, the operator $D_{m}$ by

$$
D_{m} \varphi=\frac{d \varphi}{d r}-\frac{m}{r} \varphi=r^{m} \frac{d}{d r}\left(r^{-m} \varphi\right) .
$$

The norm of $\mathbf{w}$ in $X$ is given by

$$
\|\mathbf{w}\|_{X}^{2}=\sum_{m \in \mathbf{Z}}\left\|D_{m} v_{m}\right\|_{L_{r}^{2}}^{2}
$$

Remark 2.1. Since $q$ belongs to $L_{0}^{2}(\Omega)$, there holds $\int_{0}^{1} r q_{0} d r=0$.

We can expand $\nabla q$ and $\Delta \mathbf{w}$ in the basis $\left(\mathbf{V}_{m}, \mathbf{W}_{m}\right)$ :

$$
\begin{aligned}
\nabla q & =\sum_{m \in \mathbf{Z}}\left(q_{m+1}^{\prime}+\frac{m+1}{r} q_{m+1}\right) \mathbf{V}_{m}+\sum_{m \in \mathbf{Z}}\left(q_{m-1}^{\prime}-\frac{m-1}{r} q_{m-1}\right) \mathbf{W}_{m}, \\
\Delta \mathbf{w} & =\sum_{m \in \mathbf{Z}}\left(\Delta_{r} v_{m}-\frac{m^{2}}{r^{2}} v_{m}\right) \mathbf{V}_{m}+\sum_{m \in \mathbf{Z}}\left(\Delta_{r} w_{m}-\frac{m^{2}}{r^{2}} w_{m}\right) \mathbf{W}_{m} .
\end{aligned}
$$

Noting that

$$
\Delta_{r}-\frac{m^{2}}{r^{2}}=\left(\frac{d}{d r}+\frac{m+1}{r}\right)\left(\frac{d}{d r}-\frac{m}{r}\right)=\left(\frac{d}{d r}-\frac{m-1}{r}\right)\left(\frac{d}{d r}+\frac{m}{r}\right),
$$

we see that $\Delta \mathbf{w}+\nabla q=\mathbf{0}$ is equivalent to

$$
\forall m \in \mathbf{Z}, \quad\left(\frac{d}{d r}+\frac{m+1}{r}\right)\left(q_{m+1}+D_{m} v_{m}\right)=0 .
$$

For any $m$ in $\mathbf{Z},(2.12)$ can be rewritten as $q_{m+1}+D_{m} v_{m}=c_{m} r^{-(m+1)}$. If $m$ is positive, $r^{-(m+1)}$ does not belong to $L_{r}^{2}$, which contradicts the fact that $\mathbf{w}$ belongs to $\mathbf{H}^{1}(\Omega)$ and $q$ to $L^{2}(\Omega)$. Hence, $c_{m}$ vanishes. For $m<0$, the constant is determined by the boundary conditions, and we finally get

$$
\begin{gathered}
m \geq 0, \quad D_{m} v_{m}=-q_{m+1} \\
m<0, \quad D_{m} v_{m}=-q_{m+1}-2 m r^{-(m+1)} \int_{0}^{1} \rho^{-m} q_{m+1}(\rho) d \rho .
\end{gathered}
$$

This can be solved in the correct spaces by

$$
\begin{gathered}
m \geq 0, \quad v_{m}=r^{m} \int_{r}^{1} \rho^{-m} q_{m+1}(\rho) d \rho \\
m<0, \quad v_{m}=-r^{m} \int_{0}^{r} \rho^{-m} q_{m+1}(\rho) d \rho+r^{-m} \int_{0}^{1} \rho^{-m} q_{m+1}(\rho) d \rho .
\end{gathered}
$$


We now express the norms:

$$
\text { For } \begin{aligned}
m \leq-2, \quad\left\|q_{m+1}\right\|_{L_{r}^{2}}^{2}-\left\|D_{m} v_{m}\right\|_{L_{r}^{2}}^{2} & =-2 m\left|\int_{0}^{1} \rho^{-m} q_{m+1}(\rho) d \rho\right|^{2} \\
& =-2 m\left|\int_{0}^{1} \rho^{-m} q_{-m-1}(\rho) d \rho\right|^{2} \\
& =-2 m\left|\int_{0}^{1} \rho^{-m} D_{-m-2} v_{-m-2}(\rho) d \rho\right|^{2}
\end{aligned}
$$

By the Cauchy-Schwarz inequality we get

$$
\begin{gathered}
\text { for } m \leq-2, \quad\left\|q_{m+1}\right\|_{L_{r}^{2}}^{2} \leq\left\|D_{m} v_{m}\right\|_{L_{r}^{2}}^{2}+\left\|D_{-m-2} v_{-m-2}\right\|_{L_{r}^{2}}^{2} ; \\
\quad \text { for } m \geq-1, \quad\left\|q_{m+1}\right\|_{L_{r}^{2}}^{2}=\left\|D_{m} v_{m}\right\|_{L_{r}^{2}}^{2},
\end{gathered}
$$

which gives

$$
\|q\|_{0}^{2} \leq 2\|\mathbf{w}\|_{X}^{2}
$$

If $q$ is given by $q_{m}=a_{m} r^{|m|} ; q_{0}=0$, then $D_{m} v_{m}$ vanishes for $m<0$, which gives equality.

\section{A Galerkin method For the Stokes PROBlem in a DisC}

Let $N$ and $K$ be two integers greater than or equal to 2 . Let $\mathbf{S}_{K}([0,2 \pi])$ be the set of trigonometric polynomials in $\theta$ of degree less than or equal to $K$, and $\mathbf{P}_{N}([0,1])$ the set of polynomials in $r$ of degree less than or equal to $N$. Before introducing the discrete spaces, let us write precisely the bilinear forms $a$ and $b$. If $u$ and $v$ are expanded in the $\left(\mathbf{V}_{m}, W_{m}\right)$ basis with coefficients $\left(v_{m}, w_{m}\right)$ and $\left(\tilde{v}_{m}, \tilde{w}_{m}\right)$, and if the coefficients of $q$ in the $\left\{H_{m}\right\}$ are denoted by $q_{m}$, we have

$$
\begin{gathered}
a(\mathbf{u}, \mathbf{v})=\frac{1}{2} \sum_{m \in \mathbf{Z}}\left[\left(D_{m-1} v_{m-1}, D_{m-1} \tilde{v}_{m-1}\right)_{r}+\left(D_{-m-1} w_{m+1}, D_{-m-1} \tilde{w}_{m+1}\right)_{r}\right], \\
b(\mathbf{v}, q)=\frac{1}{2} \sum_{m \in \mathbf{Z}}\left(q_{m}, D_{m-1} \tilde{v}_{m-1}+D_{-m-1} \tilde{w}_{m+1}\right)_{r} .
\end{gathered}
$$

This suggests to choose $q$ in $\mathbf{S}_{N-1} \otimes \mathbf{P}_{N-1}$, and $\mathbf{v}$ in such a space that $D_{m-1} \tilde{v}_{m-1}$ vanishes for $|m-1| \geq N-1$, and belongs to $\mathbf{P}_{N-1}$. This introduces a term in $\ln r$, which cannot be avoided.

The discrete space $M_{N}$ is defined by

$$
M_{N}=M \cap\left(\mathbf{S}_{N-1} \otimes \mathbf{P}_{N-1}\right),
$$

where $M=L_{0}^{2}(\Omega)$. Any $q$ in $\mathbf{S}_{N-1} \otimes \mathbf{P}_{N-1}$ is expanded in separate variables as

$$
q=\sum_{|m| \leq N-1} q_{m} H_{m} ; \quad q_{m} \in \mathbf{P}_{N-1},
$$

and the discrete space $X_{N}$ is defined by

$$
X_{N}=X \cap H_{N},
$$

where $X=\mathbf{H}_{0}^{1}(\Omega)$ and $H_{N}$ is the space of real functions $\mathbf{v}$ such that

$$
\mathbf{v}=\sum_{m=-N}^{N-2} v_{m} \mathbf{V}_{m}+\sum_{m=-(N-2)}^{N} w_{m} \mathbf{W}_{m}
$$


SPECTRAL METHODS IN POLAR COORDINATES FOR THE STOKES PROBLEM 513

where $v_{m}$ belongs to $\mathbf{P}_{N}$ for $-N \leq m \leq 0$, and to $\mathbf{P}_{N} \oplus \mathbb{Q}_{m}$ for $1 \leq m \leq N-2$, where $\mathbb{Q}_{m}=\left\{g(r): g(r)=c r^{m} \ln r, c \in \mathbb{C}\right\}$. We provide $X_{N}$ and $M_{N}$ with the inner products of $X$ and $M$. The discrete problem is the following: find $\left(\mathbf{u}_{N}, p_{N}\right)$ in $X_{N} \times M_{N}$ such that

$$
\begin{cases}\forall \mathbf{v} \in X_{N}, & a\left(\mathbf{u}_{N}, \mathbf{v}\right)+b\left(\mathbf{v}, p_{N}\right)=L(\mathbf{v}), \\ \forall q \in M_{N}, & b\left(\mathbf{u}_{N}, q\right)=0 .\end{cases}
$$

In order to analyze this problem, we need some classical results on Jacobi polynomials. The definitions and results can be found in [1] or [6].

3.1. Jacobi polynomials on $[0,1]$. Let $\omega$ be a positive function on $[0,1]$ such that, for any $k \geq 0, \omega r^{k}$ is integrable. Define

$$
L_{\omega}^{2}(0,1)=\left\{v \in \mathcal{D}^{\prime}(0,1), \int_{0}^{1} \omega(r)|v|^{2}(r) d r<+\infty\right\}
$$

and provide this with the natural scalar product $(v, w)_{\omega}=\int_{0}^{1} \omega(r) v(r) \bar{w}(r) d r$ and the corresponding norm $\|\cdot\|_{L_{\omega}^{2}}$. For any given weight $\omega$, there exists a sequence of orthogonal polynomials in $L_{\omega}^{2}(0,1)$. If $\alpha, \beta$ are two integers, and $\omega=\omega_{\alpha, \beta}=$ $(1-r)^{\alpha} r^{\beta}$, they are the sequence of Jacobi polynomials $J_{n}^{\alpha, \beta}$, normalized by $J_{n}^{\alpha, \beta}(1)=\left(\begin{array}{c}n+\alpha \\ n\end{array}\right)$. Their norm is given by

$$
\left\|J_{n}^{\alpha, \beta}\right\|_{L_{\omega_{\alpha, \beta}^{2}}^{2}}^{2}=\frac{(n+\alpha) !(n+\beta) !}{(n+\alpha+\beta) !(2 n+\alpha+\beta+1) n !} .
$$

The unbounded operator $L_{\alpha, \beta}$ on $L_{\omega_{\alpha, \beta}}^{2}(0,1)$ is defined by

$$
L_{\alpha, \beta}=-\frac{1}{\omega_{\alpha, \beta}} \frac{d}{d r}\left[\omega_{\alpha+1, \beta+1} \frac{d}{d r}\right] .
$$

It is selfadjoint positive on $L_{\omega_{\alpha, \beta}}^{2}(0,1)$, the eigenfunctions are $J_{n}^{\alpha, \beta}$ with eigenvalues $\lambda_{n}^{\alpha, \beta}=n(n+\alpha+\beta+1)$. The polynomials $J_{n}^{\alpha, \beta}$ satisfy the differential equation

$$
r(1-r)\left(J_{n}^{\alpha, \beta}\right)^{\prime \prime}+(\beta+1-(\alpha+\beta+2) r)\left(J_{n}^{\alpha, \beta}\right)^{\prime}+\lambda_{n}^{\alpha, \beta} J_{n}^{\alpha, \beta}=0 .
$$

Moreover, the Jacobi polynomials are given by the recursion formula

$$
\begin{gathered}
2(n+1)(n+\alpha+\beta+1)(2 n+\alpha+\beta) J_{n+1}^{\alpha, \beta} \\
=(2 n+\alpha+\beta+1)\left[\alpha^{2}-\beta^{2}+(2 n+\alpha+\beta+2)(2 n+\alpha+\beta)(2 r-1)\right] J_{n}^{\alpha, \beta} \\
-2(n+\alpha)(n+\beta)(2 n+\alpha+\beta+2) J_{n-1}^{\alpha, \beta}, \\
J_{0}^{\alpha, \beta}=1 ; \quad J_{1}^{\alpha, \beta}=(\alpha+\beta+2) r-(\beta+1) .
\end{gathered}
$$

We shall use the formula relating $J_{n}^{\alpha, \beta}$ and $J_{n-1}^{\alpha+1, \beta+1}$ :

$$
\frac{d}{d r} J_{n}^{\alpha, \beta}=(n+\alpha+\beta+1) J_{n-1}^{\alpha+1, \beta+1}
$$

and several easy results on $J_{n}^{0,1}$ and $J_{n}^{1,1}$. The sequence $J_{n}^{0,1}$ is orthogonal for the weight $r$. One has

$$
J_{n}^{0,1}(0)=(-1)^{n}(n+1)
$$


and (by integration of $(3.8)$ on $[0,1])$

$$
\int_{0}^{1} J_{n}^{0,1}(r) d r=2(-1)^{n}\left\|J_{n}^{0,1}\right\|_{L_{\omega_{0,1}}^{2}}^{2}
$$

The polynomials $J_{n}^{0,1}$ and $J_{n}^{0,0}$ (the $n$th Legendre polynomial) are related by

$$
J_{n}^{0,1}=\frac{J_{n}^{0,0}+J_{n+1}^{0,0}}{2 r} .
$$

3.2. Existence and uniqueness: the discrete inf-sup condition. Since $X_{N}$ and $M_{N}$ are subspaces of $X$ and $M$, properties (i), (ii) and (iv) in Theorem B are satisfied. For existence and uniqueness, we merely need to prove (iii). We shall prove the constant in (iii) to be the same as in the continuous case.

Theorem 3.1. On $X_{N} \times M_{N}$ one has the uniform inf-sup condition

$$
\inf _{q \in M_{N}} \sup _{\mathbf{v} \in X_{N}} \frac{b(\mathbf{v}, q)}{\|\mathbf{v}\|_{X}\|q\|_{M}}=\frac{1}{\sqrt{2}}
$$

Proof. Again, we have

$$
\inf _{q \in M_{N}} \sup _{\mathbf{v} \in X_{N}} \frac{b(\mathbf{v}, q)}{\|\mathbf{v}\|_{X}\|q\|_{M}}=\inf _{q \in M_{N}} \frac{\|\mathbf{w}\|_{X}}{\|q\|_{M}}
$$

where $\mathbf{w}$ is the unique solution of

$$
\forall \mathbf{v} \in X_{N}, \quad(\Delta \mathbf{w}+\nabla q, \mathbf{v})_{0}=0 .
$$

Using formula (2.14a), we can easily see that if $q$ belongs to $M_{N}$, then $\mathbf{w}=$ $-(\Delta)^{-1} \nabla q$ belongs to $X_{N}$. The constant is thus greater than or equal to $\frac{1}{\sqrt{2}}$. The choice $q_{0}=0, q_{m}=r^{m}, 1 \leq m \leq N-1$, gives equality.

This result, together with Theorem A, leads to the conclusion:

Theorem 3.2. For any $\mathbf{f}$ in $H^{-1}(D(0,1))$, problem (3.5) has a unique solution $\left(\mathbf{u}_{N}, p_{N}\right)$ in $X_{N} \times M_{N}$, and

$$
\left\|\mathbf{u}_{N}\right\|_{X}^{2}+\left\|p_{N}\right\|_{0}^{2} \leq C\|\mathbf{f}\|_{-1}^{2}
$$

3.3. Projection in weighted spaces on $[0,1]$. In order to obtain error estimates, we need one-dimensional projection results in weighted Sobolev spaces on $[0,1]$. Results of the same type have been obtained in [1] for the weights $\omega_{\alpha, \alpha}$; our proofs rely in a large part on their methods. The additional difficulties come from the fact that we cannot use any Hardy inequality.

For any positive integer $m$, we denote $H_{r}^{p}(0,1)=\left\{\psi \in \mathcal{D}^{\prime}(0,1), \psi^{(j)} \in L_{r}^{2}(0,1)\right.$ for any $j, 0 \leq j \leq p\}$ and furnish it with the norm $\|\psi\|_{H_{r}^{p}}^{2}=\sum_{0 \leq j \leq p}\left\|\psi^{(j)}\right\|_{L_{r}^{2}}^{2}$.

Theorem 3.3. (i) For any $q$ in $L_{r}^{2}$, there exists a unique polynomial $\Pi_{N} q$ in $\mathbf{P}_{N}$ such that

$$
\forall Q \in \mathbf{P}_{N}, \quad\left\|q-\Pi_{N} q\right\|_{L_{r}^{2}} \leq\|q-Q\|_{L_{r}^{2}} .
$$

(ii) For any positive integer $p$, one has

$$
\forall q \in H_{r}^{p}, \quad\left\|q-\Pi_{N} q\right\|_{L_{r}^{2}} \leq C N^{-p}\|q\|_{H_{r}^{p}} .
$$


SPECTRAL METHODS IN POLAR COORDINATES FOR THE STOKES PROBLEM 515 Proof. Expand $q$ in the polynomials $Q_{n}=J_{n}^{0,1}$ :

$$
q=\sum_{n=0}^{\infty} q_{n} Q_{n} ; \quad q_{n}=\frac{\left(q, Q_{n}\right)_{r}}{\left\|Q_{n}\right\|_{L_{r}^{2}}^{2}} .
$$

Then $\Pi_{N} q$ is given by

$$
\Pi_{N} q=\sum_{n=0}^{N} q_{n} Q_{n},
$$

and

$$
\left\|q-\Pi_{N} q\right\|_{L_{r}^{2}}^{2}=\sum_{n=N+1}^{\infty}\left|q_{n}\right|^{2}\left\|Q_{n}\right\|_{L_{r}^{2}}^{2} .
$$

Here, $Q_{n}$ is an eigenfunction of the selfadjoint operator $L=L_{0,1}$, corresponding to the eigenvalue $\lambda_{n}=\lambda_{n}^{0,1}=n(n+2)$. Then, for any integer $s$,

$$
\begin{gathered}
\left(q, Q_{n}\right)_{r}=\frac{1}{\lambda_{n}^{s}}\left(L^{s} q, Q_{n}\right)_{r}, \\
\left\|q-\Pi_{N} q\right\|_{L_{r}^{2}}^{2}=\sum_{n=N+1}^{\infty} \frac{1}{\lambda_{n}^{2 s}} \frac{\left[\left(L^{s} q, Q_{n}\right)_{r}\right]^{2}}{\left\|Q_{n}\right\|_{L_{r}^{2}}^{2}},
\end{gathered}
$$

which gives, for $q$ sufficiently smooth, the bounds

(3.24) $\left\|q-\Pi_{N} q\right\|_{L_{r}^{2}}^{2} \leq \frac{C}{N^{4 s}}\left\|L^{s} q\right\|_{L_{r}^{2}}^{2}, \quad\left\|q-\Pi_{N} q\right\|_{L_{r}^{2}}^{2} \leq \frac{C}{N^{4 s+2}}\left|\left(L^{s} q, L^{s+1} q\right)_{r}\right|$.

In order to estimate $\left\|L^{s} q\right\|_{L_{r}^{2}}^{2}$ and $\left(L^{s} q, L^{s+1} q\right)_{r}$, we introduce, for any integer $k$ and any function $q$ defined on $[0,1]$, the quantities

$$
\left\|\left.|| q\left|\|_{k}^{2}=\sum_{j=0}^{k} \int_{0}^{1} r^{j+1}(1-r)^{j}\right| q^{(j)}\right|^{2} d r .\right.
$$

If $q$ belongs to $H_{r}^{k}$, then $\||q|\|_{k}^{2}$ is well defined and $\||q|\|_{k}^{2} \leq\|q\|_{H_{r}^{k}}^{2}$. It is easy to see by induction that for any positive integer $s$, one has the following bounds:

$$
\begin{aligned}
& \forall q \in H_{r}^{2 s}, \quad\left\|L^{s} q\right\|_{L_{r}^{2}} \leq\left\|\mid L^{s} q\right\|\left\|_{0} \leq C\right\|\|q\|_{2 s} ; \\
& \forall q \in H_{r}^{2 s+1}, \quad\left(L^{s} q, L^{s+1} q\right)_{r} \leq\left\|\mid L^{s} q\right\|\left\|_{1} \leq C\right\|\|q\|_{2 s+1} .
\end{aligned}
$$

This ends the proof of the theorem.

The results in $H_{r}^{1}$ are less classical. Let $\widetilde{\mathbf{P}}_{N}([0,1])$ be the subspace of $\mathbf{P}_{N}([0,1])$ of polynomials vanishing at $r=1$.

Theorem 3.4. (i) For any $q$ in $H_{r}^{1} \cap \mathcal{C}^{0}([0,1])$, there exists a unique polynomial $\Pi_{N}^{1} q$ in $\mathbf{P}_{N}$ such that

$$
\Pi_{N}^{1} q(1)=q(1) ; \quad \forall Q \in \widetilde{\mathbf{P}}_{N}, \quad\left(\left(q-\Pi_{N}^{1} q\right)^{\prime}, Q^{\prime}\right)_{r}=0 .
$$

(ii) For any $q$ in $H_{r}^{p}$ with $p \geq 2$,

$$
\begin{gathered}
\left\|\left(q-\Pi_{N}^{1} q\right)^{\prime}\right\|_{L_{r}^{2}} \leq C N^{-(p-1)}\|q\|_{H_{r}^{p}} \\
\left|\left(q-\Pi_{N}^{1} q\right)(0)\right| \leq C N^{-(p-1)}\|q\|_{H_{r}^{p}}
\end{gathered}
$$


Proof. (i) Since $q^{\prime}$ belongs to $L_{r}^{2}$, its projection $\Pi_{N-1} q^{\prime}$ on $\mathbf{P}_{N-1}$ is well defined. Then $\Pi_{N}^{1} q$ is uniquely determined by

$$
\Pi_{N}^{1} q=q(1)-\int_{r}^{1} \Pi_{N-1} q^{\prime}(\rho) d \rho .
$$

(ii) Assume now that $q$ belongs to $H_{r}^{p}$ for $p \geq 2$. According to [14], $q$ belongs to $\mathcal{C}^{0}([0,1])$, so $\Pi_{N}^{1} q$ is well defined, and

$$
\left\|\left(q-\Pi_{N}^{1} q\right)^{\prime}\right\|_{L_{r}^{2}}=\left\|q^{\prime}-\Pi_{N-1} q^{\prime}\right\|_{L_{r}^{2}}
$$

which, together with (3.18) proves (3.27).

In order to prove (3.28), we write

$$
\left(q-\Pi_{N}^{1} q\right)(0)=\int_{0}^{1}\left(q^{\prime}-\Pi_{N-1} q^{\prime}\right)(\rho) d \rho
$$

and proceed as in Theorem 3.3: expand $q^{\prime}$ in the polynomials $Q_{n}: q^{\prime}=\sum_{n=0}^{\infty} q_{n} Q_{n}$, and

$$
\int_{0}^{1}\left(q^{\prime}-\Pi_{N-1} q^{\prime}\right)(\rho) d \rho=\sum_{n=N}^{+\infty} q_{n} \int_{0}^{1} Q_{n}(\rho) d \rho=2 \sum_{n=N}^{+\infty}(-1)^{n} q_{n}\left\|Q_{n}\right\|_{L_{r}^{2}}^{2}
$$

(using (3.12)). Then, for any $s \geq 0$,

$$
\int_{0}^{1}\left(q^{\prime}-\Pi_{N-1} q^{\prime}\right)(\rho) d \rho=2 \sum_{n=N}^{+\infty} \frac{(-1)^{n}}{\lambda_{n}^{s}}\left(L^{s} q^{\prime}, Q_{n}\right)_{r},
$$

and by the Cauchy-Schwarz inequality,

$$
\left|\left(q-\Pi_{N}^{1} q\right)(0)\right|^{2} \leq 4\left(\sum_{n=N}^{+\infty} \frac{\left[\left(L^{s} q^{\prime}, Q_{n}\right)_{r}\right]^{2}}{\left\|Q_{n}\right\|_{L_{r}^{2}}^{2}}\right)\left(\sum_{n=N}^{+\infty} \frac{\left\|Q_{n}\right\|_{L_{r}^{2}}^{2}}{\lambda_{n}^{2 s}}\right) .
$$

The first term has been estimated in Theorem 3.3. As for the second, we have

$$
2 \sum_{n=N}^{+\infty} \frac{\left\|Q_{n}\right\|_{L_{r}^{2}}^{2}}{\lambda_{n}^{2 s}} \leq \sum_{n=N}^{+\infty} \frac{1}{n^{4 s+1}}
$$

and for $s>0$ (cf. [7])

$$
\sum_{n=N}^{+\infty} \frac{1}{n^{4 s+1}} \sim \int_{N}^{+\infty} x^{-(4 s+1)} d x=\frac{1}{4 s} N^{-4 s}
$$

This, together with (3.25a), gives the successive bounds:

$$
\forall s>0, \quad\left|\left(q-\Pi_{N}^{1} q\right)(0)\right| \leq C N^{-2 s}\left\|L^{s} q^{\prime}\right\|_{L_{r}^{2}} \leq C N^{-2 s}\left\|q^{\prime}\right\|_{H_{r}^{2 s}} \leq C N^{-2 s}\|q\|_{H_{r}^{2 s+1}} .
$$

In the same way, for any $s \geq 0$, one has

$$
\begin{aligned}
\left|\left(q-\Pi_{N}^{1} q\right)(0)\right|^{2} & \leq C N^{-4 s-2}\left|\left(L^{s} q^{\prime}, L^{s+1} q^{\prime}\right)_{r}\right| \\
& \leq C N^{-4 s-2}\left\|q^{\prime}\right\|_{H_{r}^{2 s+1}}^{2} \leq C N^{-4 s-2}\|q\|_{H_{r}^{2 s+2}}^{2},
\end{aligned}
$$

which gives (3.28) for any integer $p \geq 2$.

We also need to estimate the norm of $q-\Pi_{N}^{1} q$ in $L_{r}^{2}$.

Theorem 3.5. For any integer $p \geq 2$, for any $q$ in $H_{r}^{p}$, one has

$$
\left\|q-\Pi_{N}^{1} q\right\|_{L_{r}^{2}} \leq C N^{-p}\|q\|_{H_{r}^{p}}
$$


SPECTRAL METHODS IN POLAR COORDINATES FOR THE STOKES PROBLEM 517

Proof. It goes by a duality argument:

$$
\left\|q-\Pi_{N}^{1} q\right\|_{L_{r}^{2}}=\sup _{g \in L_{r}^{2}} \frac{\left(q-\Pi_{N}^{1} q, g\right)_{r}}{\|g\|_{L_{r}^{2}}} .
$$

We shall use a bidimensional result: if $\Omega$ is smooth enough, we know (see for example [3]) that for any $f$ in $L^{2}(\Omega)$, there exists a unique $u$ in $H_{0}^{1}(\Omega)$ such that $-\Delta u=f$. Furthermore $u$ belongs to $H^{2}(\Omega)$ and $\|u\|_{2} \leq C\|f\|_{0}$. Here, $\Omega$ is $D(0,1)$, and we choose $f=g(r)$ in $L_{r}^{2}$. Then $u=\psi(r)$ belongs to $H_{r}^{2}$ and is such that

$$
\begin{gathered}
-\frac{1}{r} \frac{d}{d r}\left(r \frac{d \psi}{d r}\right)=g, \\
\|\psi\|_{H_{r}^{2}} \leq C\|g\|_{L_{r}^{2}} .
\end{gathered}
$$

Integration by parts, using the boundary data, gives

$$
\left(q-\Pi_{N}^{1} q, g\right)_{r}=\left(\left(q-\Pi_{N}^{1} q\right)^{\prime}, \psi^{\prime}\right)_{r} .
$$

Moreover, since $\psi(1)=0$, we have that $\Pi_{N}^{1} \psi$ belongs to $\widetilde{\mathbf{P}}_{N}$ and

$$
\left(\left(q-\Pi_{N}^{1} q\right)^{\prime},\left(\Pi_{N}^{1} \psi\right)^{\prime}\right)_{r}=0 .
$$

Hence,

$$
\left(q-\Pi_{N}^{1} q, g\right)_{r}=\left(\left(q-\Pi_{N}^{1} q\right)^{\prime},\left(\psi-\Pi_{N}^{1} \psi\right)^{\prime}\right)_{r} \leq\left\|\left(q-\Pi_{N}^{1} q\right)^{\prime}\right\|_{L_{r}^{2}}\left\|\left(\psi-\Pi_{N}^{1} \psi\right)^{\prime}\right\|_{L_{r}^{2}} .
$$

Using (3.27) and (3.33), we have

$$
\begin{gathered}
\left\|\left(\psi-\Pi_{N}^{1} \psi\right)^{\prime}\right\|_{L_{r}^{2}} \leq C N^{-1}\|\psi\|_{H_{r}^{2}} \leq C\|g\|_{L_{r}^{2}}, \\
\left\|\left(q-\Pi_{N}^{1} q\right)^{\prime}\right\|_{L_{r}^{2}} \leq C N^{-(p-1)}\|q\|_{H_{r}^{p}},
\end{gathered}
$$

and for any $g$ in $L_{r}^{2}$,

$$
\left(q-\Pi_{N}^{1} q, g\right)_{r} \leq C N^{-p}\|q\|_{H_{r}^{p}}\|g\|_{L_{r}^{2}} .
$$

We now plug (3.34) in (3.31) and get (3.30).

3.4. Approximation results. Because of the ellipticity of the bilinear form $a$ and the uniform inf-sup condition, Theorem 1.1 in [8] gives a first approximation result: if $(\mathbf{u}, p)$ and $\left(\mathbf{u}_{N}, p_{N}\right)$ are the solutions to (2.3) and (3.5), respectively, there exists a positive constant $C$ such that

$$
\left\|\mathbf{u}-\mathbf{u}_{N}\right\|_{X}+\left\|p-p_{N}\right\|_{0} \leq C\left\{\inf _{\mathbf{v}_{N} \in V_{N}}\left\|\mathbf{u}-\mathbf{v}_{N}\right\|_{X}+\inf _{q_{N} \in M_{N}}\left\|p-q_{N}\right\|_{0}\right\},
$$

where $V_{N}$ is the discrete space corresponding to $V$ :

$$
V_{N}=\left\{\mathbf{v}_{N} \in X_{N}, \forall q_{N} \in M_{N}, b\left(\mathbf{v}_{N}, q_{N}\right)=0\right\} .
$$

It remains to estimate the expressions in the right-hand side of (3.35).

Theorem 3.6. Let $p$ be an integer greater than or equal to zero. For any $q$ in $M \cap H^{p}(\Omega)$, its projection $\tilde{q}=\Pi_{N, K} q$ on $\mathrm{S}_{K} \otimes \mathbf{P}_{N}$ belongs to $M$ and satisfies the following estimate:

$$
\|q-\tilde{q}\|_{0} \leq C \min \left\{\frac{1}{N^{p}}, \frac{1}{K^{p}}\right\}\|q\|_{p} .
$$

On the other hand, $\mathbf{u}$ belongs to $V$. It can be approximated in $V \cap X_{N}$ : 
Theorem 3.7. Let $p$ be a positive integer. For any $\mathbf{v}$ in $V \cap \mathbf{H}^{p}(\Omega)$, there exists $\mathbf{w}$ in $V \cap X_{N}$ such that

$$
\|\mathbf{v}-\mathbf{w}\|_{X} \leq C N^{1-p}\|\mathbf{v}\|_{p}
$$

These two theorems give the final optimal estimate:

Theorem 3.8. Let $p$ be an integer greater than or equal to zero. If $\mathbf{f}$ belongs to $\mathbf{H}^{p}(\Omega)$, the solutions $(\mathbf{u}, p)$ and $\left(\mathbf{u}_{N}, p_{N}\right)$ to (2.3) and (3.5) satisfy the following estimate:

$$
\left\|\mathbf{u}-\mathbf{u}_{N}\right\|_{X}+\left\|p-p_{N}\right\|_{0} \leq C N^{-1-p}\|\mathbf{f}\|_{p}
$$

Proof of Theorem 3.6. We expand $q$ in $\left\{H_{k}\right\}$ as $q=\sum_{k \in \mathbf{Z}} q_{k}(r) H_{k}$. Its projection $\widetilde{\Pi}_{K}$ on $S_{K} \otimes L_{r}^{2}$ is given by $\widetilde{\Pi}_{K} q=\sum_{|k| \leq K} q_{k}(r) H_{k}$, and the operator $\Pi_{N, K}$ is defined by

$$
\tilde{q}=\Pi_{N, K} q=\sum_{|k| \leq K} \Pi_{N} q_{k}(r) H_{k} .
$$

In particular, if $q$ belongs to $M$, then $\tilde{q}$ belongs to $M$, and

$$
\|q-\tilde{q}\|_{0} \leq\left\|q-\widetilde{\Pi}_{K} q\right\|_{0}+\left\|\widetilde{\Pi}_{K} q-\tilde{q}\right\|_{0}
$$

The first term is estimated through the one-dimensional result in [5]:

which gives

$$
\left\|q-\widetilde{\Pi}_{K} q\right\|_{0} \leq C K^{-p}\left\|\frac{\partial^{p} q}{\partial \theta^{p}}\right\|_{0},
$$

$$
\left\|q-\widetilde{\Pi}_{K} q\right\|_{0} \leq C K^{-p}\|q\|_{p}
$$

As for the second term, we have

$$
\left\|\widetilde{\Pi}_{K} q-\tilde{q}\right\|_{0}^{2}=\sum_{|k| \leq K}\left\|q_{k}-\Pi_{N} q_{k}\right\|_{L_{r}^{2}}^{2}
$$

and using Theorem 3.3, we get

$$
\begin{gathered}
\left\|\widetilde{\Pi}_{K} q-\tilde{q}\right\|_{0}^{2} \leq C N^{-2 p} \sum_{|k| \leq K}\left\|q_{k}\right\|_{H_{r}^{p}}^{2}, \\
\left\|\widetilde{\Pi}_{K} q-\tilde{q}\right\|_{0} \leq C N^{-p}\|q\|_{p} .
\end{gathered}
$$

Plugging (3.41) and (3.42) in (3.40) gives the desired estimate in the theorem.

Proof of Theorem 3.7. Recall that for $\mathbf{v}$ in $H^{p}(\Omega) \cap H_{0}^{1}(\Omega)$, its curl $\nabla \wedge \mathbf{v}$ belongs to $H^{p-1}(\Omega)$, and

$$
\|\nabla \wedge \mathbf{v}\|_{p-1} \leq 2\|\mathbf{v}\|_{p}
$$

If moreover $\mathbf{v}$ belongs to $V$, then $\|\mathbf{v}\|_{X}=\|\nabla \wedge \mathbf{v}\|_{0}$. Here, $\nabla \wedge \mathbf{v}$ can be projected on $\mathbf{S}_{N-1} \otimes \mathbf{P}_{N-1}$ in the following way. We expand $\nabla \wedge \mathbf{v}$ and $\nabla \cdot \mathbf{v}$ in $\left\{H_{m}(\theta)\right\}$ :

$$
\begin{gathered}
\nabla \cdot \mathbf{v}=\sum_{m \in \mathbb{Z}} D_{m} v_{m} H_{m+1}+\sum_{m \in \mathbb{Z}} D_{-m} w_{m} H_{m-1}, \\
\nabla \wedge \mathbf{v}=i\left(\sum_{m \in \mathbb{Z}} D_{m} v_{m} H_{m+1}-\sum_{m \in \mathbb{Z}} D_{-m} w_{m} H_{m-1}\right) .
\end{gathered}
$$


SPECTRAL METHODS IN POLAR COORDINATES FOR THE STOKES PROBLEM 519

If $\mathbf{v}$ belongs to $V$, the function $\varphi$ defined by $\varphi=-\frac{i}{2} \nabla \wedge \mathbf{v}$ belongs to $L^{2}(\Omega)$, and by Theorem 3.6, one has the following error estimate on its projection on $\mathbf{S}_{N-1} \otimes \mathbf{P}_{N-1}, \chi=\sum_{m=-N}^{N-2} \chi_{m} H_{m+1}$,

$$
\|\varphi-\chi\|_{0} \leq c N^{1-p}\|\varphi\|_{p-1}
$$

We can define $\mathbf{w}$ in $V \cap X_{N}$ such that $\nabla \wedge \mathbf{w}=2 i \chi$. It is given by

$$
\mathbf{w}=\sum_{m=-N}^{N-2} \tilde{v}_{m} \mathbf{V}_{m}+\sum_{m=-(N-2)}^{N} \tilde{w}_{m} \mathbf{W}_{m}
$$

the coefficients being defined for $-(N-2) \leq m \leq N$ by $\tilde{w}_{m}=\overline{\tilde{v}}_{m}$, and

$$
\begin{gathered}
*-N \leq m \leq-1, \quad \tilde{v}_{m}=r^{m} \int_{0}^{r} \rho^{-m} \chi_{m}(\rho) d \rho, \\
* \tilde{v}_{0}=-\int_{r}^{1} \chi_{0}(\rho) d \rho \\
* 1 \leq m \leq N-2, \quad \tilde{v}_{m}=-r^{m} \int_{r}^{1} \rho^{-m} \chi_{m}(\rho) d \rho .
\end{gathered}
$$

The assumptions we made force $\mathbf{w}$ to belong to $X_{N} \cap V$. Moreover,

$$
\begin{gathered}
\|\mathbf{v}-\mathbf{w}\|_{X}=\|\nabla \wedge \mathbf{v}-\nabla \wedge \mathbf{w}\|_{0}=2\|\varphi-\chi\|_{0} \leq C N^{1-p}\|\varphi\|_{p-1}, \\
\|\mathbf{v}-\mathbf{w}\|_{X} \leq C N^{1-p}\|\mathbf{v}\|_{p} .
\end{gathered}
$$

There is also an $L^{2}$-estimate on the velocity:

Theorem 3.9. Let $p$ be a positive integer. If $\mathbf{f}$ belongs to $\mathbf{H}^{p}(\Omega)$, there exists a positive constant $C$ such that

$$
\left\|\mathbf{u}-\mathbf{u}_{N}\right\|_{0} \leq C N^{-2-p}\|\mathbf{f}\|_{p} .
$$

The proof is classical and will be omitted (cf. [8]).

\section{Pseudospectral method}

We start with a description of the quadrature formula we shall use in the $r$ variable.

4.1. Discrete formulation. The fully discrete formulation relies on the GaussLobatto quadrature formula for the weight $r$ on $[0,1]$ (cf. [6]).

Theorem 4.1. Let $N$ be an integer $\geq 2, r_{0}=0, r_{N}=1$. There exists a unique set of $N-1$ points $r_{j}$ in $(0,1)$ and $N-1$ positive weights $\rho_{j}$ such that

$$
\forall g \in \mathbf{P}_{2 N-1}, \quad \int_{0}^{1} r g(r) d r=\sum_{j=0}^{N} \rho_{j} g\left(r_{j}\right) .
$$

The points $r_{j}$ are the zeros of $\left(J_{N}^{0,1}\right)^{\prime}=J_{N-1}^{1,2}$, the weights $\rho_{j}$ are given by

$$
1 \leq j \leq N, \quad \rho_{j}=\frac{1}{N(N+2)\left[J_{N}^{0,1}\left(r_{j}\right)\right]^{2}}, \quad \rho_{0}=\frac{2}{N(N+2)(N+1)^{2}} .
$$


We now introduce on $C^{0}([0,1])$ the discrete inner product

$$
(f, g)_{N}=\sum_{j=0}^{N} \rho_{j} f\left(r_{j}\right) \bar{g}\left(r_{j}\right)
$$

The corresponding Hermitian form is denoted by $|\cdot|_{N}$.

Theorem 4.2. For any integer $N \geq 2,|\cdot|_{N}$ is a norm on $\mathbf{P}_{N}([0,1])$, which is equivalent to $\|\cdot\|_{L_{r}^{2}}$. More precisely, one has

$$
\forall \varphi \in \mathbf{P}_{N}([0,1]), \quad\|\varphi\|_{L_{r}^{2}} \leq|\varphi|_{N} \leq \sqrt{3}\|\varphi\|_{L_{r}^{2}}
$$

Proof. Expand $\varphi$ in $\mathbf{P}_{N}([0,1])$ in the $J_{k}^{0,1}, \varphi=\sum_{0}^{N} \lambda_{k} J_{k}^{0,1}$, express $\|\varphi\|_{L_{r}^{2}}^{2}$ and $|\varphi|_{N}^{2}$, use the fact that the continuous and discrete integration formulae agree on $\mathbf{P}_{2 N-1}$, and formula (4.2) (for details see [1] for the method and [9] in this case).

The discrete inner product is now defined in $L^{2}(\Omega)$ by

$$
(\varphi, \chi)_{N, N}=\int_{0}^{2 \pi}(\varphi(\cdot, \theta), \chi(\cdot, \theta))_{N} d \theta .
$$

Since we use trigonometric polynomials, we do not discretize the tangential integral. The discrete bilinear forms $a_{N}$ and $b_{N}$ are given by

$$
\left\{\begin{array}{l}
a_{N}(\mathbf{u}, \mathbf{v})=(\nabla \mathbf{u}, \nabla \mathbf{v})_{N, N} \\
b_{N}(\mathbf{v}, q)=-(q, \nabla \cdot \mathbf{v})_{N, N}
\end{array}\right.
$$

which can be rewritten by expanding $u$ and $v$ in the $\left(\mathbf{V}_{m}, W_{m}\right)$ basis with coefficients $\left(v_{m}, w_{m}\right)$ and $\left(\tilde{v}_{m}, \tilde{w}_{m}\right)$, and $q$ in the $\left\{H_{m}\right\}$ with coefficients $q_{m}$,

$$
\begin{aligned}
a_{N}(\mathbf{u}, \mathbf{v})=\frac{1}{2} \sum_{m=-(N-1)}^{N-1}\left[\left(D_{m-1} v_{m-1}, D_{m-1} \tilde{v}_{m-1}\right)_{N}\right. \\
\left.+\left(D_{-m-1} w_{m+1}, D_{-m-1} \tilde{w}_{m+1}\right)_{N}\right], \\
b_{N}(\mathbf{v}, q)=\frac{1}{2} \sum_{m=-(N-1)}^{N-1}\left(q_{m}, D_{m-1} \tilde{v}_{m-1}+D_{-m-1} \tilde{w}_{m+1}\right)_{N} .
\end{aligned}
$$

The special form of our discrete spaces allows the following pleasant result:

Lemma 4.1. The discrete forms $a_{N}$ and $b_{N}$ are exact on $X_{N} \times M_{N}$, i.e.,

$$
\begin{aligned}
& \forall(\mathbf{u}, \mathbf{v}) \in X_{N} \times X_{N}, \quad a_{N}(\mathbf{u}, \mathbf{v})=a(\mathbf{u}, \mathbf{v}), \\
& \forall(\mathbf{v}, q) \in X_{N} \times M_{N}, \quad b_{N}(\mathbf{v}, q)=b(\mathbf{v}, q) .
\end{aligned}
$$

The proof is straightforward and will be omitted.

The discrete problem now reads: find $\left(\tilde{\mathbf{u}}_{N}, \tilde{p}_{N}\right)$ in $X_{N} \times M_{N}$ such that

$$
\begin{cases}\forall \mathbf{v} \in X_{N}, & a_{N}\left(\tilde{\mathbf{u}}_{N}, \mathbf{v}\right)+b_{N}\left(\mathbf{v}, \tilde{p}_{N}\right)=L_{N}(\mathbf{v}), \\ \forall q \in M_{N}, & b_{N}\left(\tilde{\mathbf{u}}_{N}, q\right)=0,\end{cases}
$$

where $L_{N}$ is defined in the following way: $\mathbf{f}$ and $\mathbf{v}$ are expanded as

$$
\mathbf{f}=\sum_{m \in \mathbf{Z}} f_{m} \mathbf{V}_{m}+\sum_{m \in \mathbf{Z}} g_{m} \mathbf{W}_{m}, \quad \mathbf{v}=\sum_{m=-N}^{N-2} v_{m} \mathbf{V}_{m}+\sum_{m=-(N-2)}^{N} w_{m} \mathbf{W}_{m} ;
$$


SPECTRAL METHODS IN POLAR COORDINATES FOR THE STOKES PROBLEM 521

then

$$
2(\mathbf{f}, \mathbf{v})=\sum_{m=-N}^{N-2}\left(f_{m}, v_{m}\right)_{r}+\sum_{m=-(N-2)}^{N}\left(g_{m}, w_{m}\right)_{r} .
$$

For $1 \leq m \leq N-2$, define an operator $R_{m}$ by

$$
R_{m} h=-r^{-m-1} \int_{0}^{r} \rho^{m+1} h(\rho) d \rho .
$$

If $h$ belongs to $L_{r}^{2}(0,1)$, then $R_{m} h$ belongs to $L_{r}^{2}(0,1)$, and

$$
\left\|R_{m} h\right\|_{L_{r}^{2}}^{2} \leq \frac{1}{4(m+1)}\|h\|_{L_{r}^{2}}^{2} .
$$

Using integration by parts, we can write

$$
\begin{aligned}
2(\mathbf{f}, \mathbf{v})= & \sum_{m=-N}^{0}\left(f_{m}, v_{m}\right)_{r}+\sum_{m=1}^{N-2}\left(R_{m} f_{m}, D_{m} v_{m}\right)_{r} \\
& +\sum_{m=0}^{N}\left(g_{m}, w_{m}\right)_{r}+\sum_{m=-(N-2)}^{-1}\left(R_{-m} g_{m}, D_{-m} w_{m}\right)_{r} .
\end{aligned}
$$

We can now define the discrete linear operator by

$$
\begin{aligned}
2 L_{N}(\mathbf{v})= & \sum_{m=-N}^{0}\left(f_{m}, v_{m}\right)_{N}+\sum_{m=1}^{N-2}\left(R_{m} f_{m}, D_{m} v_{m}\right)_{N} \\
& +\sum_{m=0}^{N}\left(g_{m}, w_{m}\right)_{N}+\sum_{m=-(N-2)}^{-1}\left(R_{-m} g_{m}, D_{-m} w_{m}\right)_{N} .
\end{aligned}
$$

Theorem 4.3. If $f$ belongs to $C^{0}(\Omega)$, problem (4.7) has a unique solution.

Proof. By Lemma 4.1, we only need to check that the mapping $\mathbf{v} \rightarrow L_{N}(\mathbf{v})$ is continuous on $X_{N}$. By Theorem 4.2 and the Cauchy-Schwarz inequality,

$$
\left|L_{N}(\mathbf{v})\right|^{2} \leq C\left[\sum_{m=-N}^{0}\left|f_{m}\right|_{N}^{2}+\sum_{m=1}^{N-2}\left|R_{m} f_{m}\right|_{N}^{2}\right]\|\mathbf{v}\|_{X}^{2} .
$$

The first sum is bounded by a constant times $\|\mathbf{f}\|_{\infty}^{2}$. As for the second, we have for any $i$,

and

$$
\left|R_{m} f_{m}\left(r_{i}\right)\right|^{2} \leq \frac{1}{2 m+2}\left\|f_{m}\right\|_{L_{r}^{2}}^{2}
$$

$$
\left|L_{N}(\mathbf{v})\right| \leq C\|\mathbf{f}\|_{\infty}\|\mathbf{v}\|_{X} .
$$

4.2. Interpolation formula on $[0,1]$. Let $h$ be an element of $H_{r}^{p}(0,1)$, for $p \geq 2$. By [14], $h$ is continuous on $[0,1]$. Then define $I_{N} h$ as the polynomial interpolating $h$ at the Gauss-Lobatto points i.e.,

$$
I_{N} h \in \mathbf{P}_{N} ; \quad \forall j, 1 \leq j \leq N, \quad I_{N} h\left(r_{j}\right)=h\left(r_{j}\right) .
$$

The aim of this section is to prove the following result:

Theorem 4.4. Let $p$ be an integer $\geq 2$. If $h$ belongs to $H_{r}^{p}$, one has

$$
\left\|h-I_{N} h\right\|_{L_{r}^{2}} \leq C N^{-p}\|h\|_{H_{r}^{p}} .
$$


The proof goes along the lines in [1]:

(i) Estimate zeros and weights in (4.1).

(ii) If $h(1)=0$, estimate $\left\|I_{N} h\right\|_{L_{r}^{2}}$ by Theorem 4.3.

(iii) Estimate $\left\|h-I_{N} h\right\|_{L_{r}^{2}}$ by the projection theorems of $\S 3.2$.

(i) Estimation of the weights. From ([16, p. 353]), one has

$$
1 \leq j \leq N-1, \quad \rho_{j} \sim \frac{\pi}{N} r_{j}^{3 / 2}\left(1-r_{j}\right)^{1 / 2} .
$$

Location of the zeros.

Lemma 4.2. For any integer $N \geq 2$, the zeros $r_{j}=\cos ^{2} \frac{\theta_{j}}{2}$ of $J_{N-1}^{1,2}$ are such that $\theta_{j} \in K_{j}$, where the intervals $K_{j}$ are defined by

$$
\begin{gathered}
1 \leq j \leq\left[\frac{N}{2}\right]-1 ; \quad K_{j}=\left(\frac{j-\frac{1}{4}}{N+\frac{3}{2}} \pi, \frac{j+1}{N+1} \pi\right), \\
{\left[\frac{N}{2}\right] \leq j \leq\left[\frac{N}{2}\right]+1 ; \quad K_{j}=\left(\frac{j-\frac{1}{4}}{N+\frac{3}{2}} \pi, \frac{j+\frac{3}{4}}{N+\frac{1}{2}} \pi\right),} \\
{\left[\frac{N}{2}\right]+1 \leq j \leq N-1 ; \quad K_{j}=\left(\frac{j}{N+2} \pi, \frac{j+\frac{3}{4}}{N+\frac{1}{2}} \pi\right) .}
\end{gathered}
$$

Proof. In ([16, p. 138]) we find the location of the zeros of the Legendre polynomials $J_{N}^{0,0}$. We now use (3.10) and (3.13) to get (4.14).

(ii) Estimation of $\left\|I_{N} h\right\|_{L_{r}^{2}}$.

Lemma 4.3. Let $p$ be an integer $\geq 2$. If $h$ belongs to $H_{r}^{p}$ and $h(1)=0$, then

$$
\left\|I_{N} h\right\|_{L_{r}^{2}}^{2} \leq C\left(\|h\|_{L_{r}^{2}}^{2}+N^{-2}\left\|h^{\prime}\right\|_{L_{r}^{2}}^{2}+N^{-4}|h(0)|^{2}\right) .
$$

Proof. From Theorem 4.3, we have

$$
\left\|I_{N} h\right\|_{L_{r}^{2}}^{2} \leq\left|I_{N} h\right|_{N}^{2}=\sum_{j=0}^{N} \rho_{j}|h|^{2}\left(r_{j}\right)
$$

If $h(1)=0$, then from $(4.2)$,

$$
\left\|I_{N} h\right\|_{L_{r}^{2}}^{2} \leq 2 N^{-4}|h(0)|^{2}+\sum_{j=1}^{N-1} \rho_{j}|h|^{2}\left(r_{j}\right)
$$

and, from (4.13),

$$
\left\|I_{N} h\right\|_{L_{r}^{2}}^{2} \leq C\left(N^{-4}|h(0)|^{2}+N^{-1} \sum_{j=1}^{N-1} r_{j}^{3 / 2}\left(1-r_{j}\right)^{1 / 2}|h|^{2}\left(r_{j}\right)\right) .
$$

Using the function $G$ defined by

$$
g(r)=r^{3 / 2}(1-r)^{1 / 2} f(r), \quad F(\theta)=h(r), \quad G(\theta)=g(r), \quad \text { with } r=\cos ^{2} \frac{\theta}{2},
$$

we can rewrite (4.13) as

$$
\left\|I_{N} h\right\|_{L_{r}^{2}}^{2} \leq C\left(N^{-4}|h(0)|^{2}+N^{-1} \sum_{j=1}^{N-1} \sup _{\theta \in K_{j}}|G(\theta)|^{2}\right) .
$$


SPECTRAL METHODS IN POLAR COORDINATES FOR THE STOKES PROBLEM 523 Assume for the time being the two following results.

Lemma 4.4. Let $p$ be an integer $\geq 2$. If $\phi$ belongs to $H_{r}^{p}$ and $\phi(1)=0$, then

$$
\int_{0}^{1} \frac{|\phi(r)|^{2}}{1-r} d r \leq \int_{0}^{1} r\left|\phi^{\prime}(r)\right|^{2} d r
$$

Lemma 4.5. Let $p$ be an integer $\geq 2$. If $h$ belongs to $H_{r}^{p}$, the function $G(\theta)$ defined in (4.17) belongs to $H^{1}(0, \pi)$ and is such that

$$
\|G\|_{L^{2}(0, \pi)}=\|h\|_{L_{r}^{2}}, \quad\left\|G^{\prime}\right\|_{L^{2}(0, \pi)} \leq C\left\|h^{\prime}\right\|_{L_{r}^{2}}
$$

Lemma 1.4 in [1] reads

$$
\sup _{\theta \in K_{j}}|G(\theta)|^{2} \leq C\left(\frac{1}{\left|K_{j}\right|}\|G\|_{L^{2}\left(K_{j}\right)}^{2}+\left|K_{j}\right|\left\|G^{\prime}\right\|_{L^{2}\left(K_{j}\right)}^{2}\right)
$$

where $|K|$ is the length of the interval $K$. Note that, for any $j, K_{j}$ and $K_{j+3}$ are disjoined. Thus, the union of intervals $K_{j}$ covers at most 3 times $(0, \pi)$. Moreover, there exists a strictly positive number $C$ such that $\left|K_{j}\right| \sim \frac{C}{N}$. These two remarks, together with (4.20) and (4.21), give (4.15).

Proof of Lemma 4.4. Since $\phi$ belongs to $H^{1}(0,1)$, we write $\phi(r)=-\int_{r}^{1} \phi^{\prime}(s) d s$, and by the Cauchy-Schwarz inequality,

$$
|\phi(r)|^{2} \leq(1-r) \int_{r}^{1}\left|\phi^{\prime}(s)\right|^{2} d s,
$$

which proves that $\frac{|\phi(r)|^{2}}{1-r}$ belongs to $L^{1}(0,1)$ and

$$
\int_{0}^{1} \frac{|\phi(r)|^{2}}{1-r} d r \leq \int_{0}^{1} \int_{r}^{1}\left|\phi^{\prime}(s)\right|^{2} d s d r=\int_{0}^{1} r\left|\phi^{\prime}(r)\right|^{2} d r .
$$

Proof of Lemma 4.5. A mere change of variables in the integral shows at once that $\|G\|_{L^{2}(0, \pi)}=\|h\|_{L_{r}^{2}}$. As for the derivative, we have

$$
\left\|G^{\prime}\right\|_{L^{2}(0, \pi)}=\int_{0}^{1} r^{2}(1-r)\left|h^{\prime}(r)\right|^{2} d r+\frac{1}{16} \int_{0}^{1} \frac{20 r-16 r^{2}-3}{1-r}|h(r)|^{2} d r .
$$

On $[0,1]$, the numerator in the second integral is bounded by a strictly positive constant, and Lemma 4.4 allows us to conclude.

(iii) Estimation of $\left\|h-I_{N} h\right\|_{L_{r}^{2}}$. For any $h$ in $H_{r}^{p}$, we introduce $\Pi_{N}^{1} h$ as in Theorem 3.4. Since it belongs to $\mathbf{P}_{N}$, we can write

$$
\left\|h-I_{N} h\right\|_{L_{r}^{2}}^{2} \leq 2\left(\left\|h-\Pi_{N}^{1} h\right\|_{L_{r}^{2}}^{2}+\left\|I_{N}\left(\Pi_{N}^{1} h-h\right)\right\|_{L_{r}^{2}}^{2}\right) .
$$

Using Lemma 4.3 for $\Pi_{N}^{1} h-h$, we get

$$
\left\|h-I_{N} h\right\|_{L_{r}^{2}}^{2} \leq C\left(\left\|\Pi_{N}^{1} h-h\right\|_{L_{r}^{2}}^{2}+N^{-2}\left\|\left(\Pi_{N}^{1} h-h\right)^{\prime}\right\|_{L_{r}^{2}}^{2}+N^{-4}\left|\left(\Pi_{N}^{1} h-h\right)(0)\right|^{2}\right) .
$$

Theorems 3.4 and 3.5 give (4.12) in Theorem 4.4 . 
4.3. Approximation results. We start with the same result as in $\S 3.4$. The solutions $(\mathbf{u}, p)$ and $\left(\tilde{\mathbf{u}}_{N}, \tilde{p}_{N}\right)$ to (2.3) and (4.7) satisfy a first estimate:

$$
\begin{aligned}
\| \mathbf{u}- & \tilde{\mathbf{u}}_{N}\left\|_{X}+\right\| p-\tilde{p}_{N} \|_{0} \\
& \leq C\left\{\inf _{\mathbf{v}_{N} \in V_{N}}\left\|\mathbf{u}-\mathbf{v}_{N}\right\|_{X}+\inf _{q_{N} \in M_{N}}\left\|p-q_{N}\right\|_{0}+\left\|L-L_{N}\right\|\right\},
\end{aligned}
$$

where $V_{N}=\left\{\mathbf{v}_{N} \in X_{N}, \forall q_{N} \in M_{N}, b_{N}\left(\mathbf{v}_{N}, q_{N}\right)=0\right\}$, and

$$
\left\|L-L_{N}\right\|=\sup _{\mathbf{v}_{N} \in V_{N}} \frac{\left|L\left(\mathbf{v}_{N}\right)-L_{N}\left(\mathbf{v}_{N}\right)\right|}{\left\|\mathbf{v}_{N}\right\|_{X}} .
$$

Using the results in $\S \S 3.3$ and 4.3, we shall prove the following result.

Theorem 4.5. Let $p$ be an integer $\geq 0$. If $f$ belongs to $H^{p}(\Omega)$, the solutions $(\mathbf{u}, p)$ and $\left(\tilde{\mathbf{u}}_{N}, \tilde{p}_{N}\right)$ to (2.3) and (4.7) satisfy the following estimates:

$$
\left\|\mathbf{u}-\tilde{\mathbf{u}}_{N}\right\|_{X}+\left\|p-\tilde{p}_{N}\right\|_{0} \leq C N^{-p}\|\mathbf{f}\|_{p}
$$

Proof. From Theorem 3.6, since $p$ belongs to $H^{p+1}(\Omega)$, we have

$$
\left\|p-\Pi_{N-1, N-1} p\right\|_{0} \leq C N^{-(p+1)}\|p\|_{p+1} \leq C N^{-(p+1)}\|\mathbf{f}\|_{p},
$$

and since $\mathbf{u}$ belongs to $V \cap \mathbf{H}^{p+2}(\Omega)$, there exists $\mathbf{v}_{N}$ in $V \cap X_{N}$ such that

$$
\left\|\mathbf{u}-\mathbf{v}_{N}\right\|_{X} \leq C N^{1-(p+2)}\|\mathbf{u}\|_{p+2} \leq C N^{-(p+1)}\|\mathbf{f}\|_{p} .
$$

It remains to estimate $\left\|L-L_{N}\right\|$. For any $\mathbf{v}$ in $X_{N}$, we have

$$
\begin{aligned}
\left|L(\mathbf{v})-L_{N}(\mathbf{v})\right| \leq & \sum_{m=-N}^{0}\left|\left(f_{m}, v_{m}\right)_{N}-\left(f_{m}, v_{m}\right)_{r}\right| \\
& +\sum_{m=1}^{N-2}\left|\left(R_{m} f_{m}, D_{m} v_{m}\right)_{N}-\left(R_{m} f_{m}, D_{m} v_{m}\right)_{r}\right| .
\end{aligned}
$$

Let us estimate the first sum. For $-N \leq m \leq 0$, since the quadrature formula is exact on $\mathbf{P}_{2 N-1}$,

$$
\left(f_{m}, v_{m}\right)_{N}-\left(f_{m}, v_{m}\right)_{r}=\left(I_{N} f_{m}-\Pi_{N-1} f_{m}, v_{m}\right)_{N}-\left(f_{m}-\Pi_{N-1} f_{m}, v_{m}\right)_{r}
$$

and by Theorem 4.2 ,

$$
\left|\left(I_{N} f_{m}-\Pi_{N-1} f_{m}, v_{m}\right)_{N}\right| \leq 3\left\|I_{N} f_{m}-\Pi_{N-1} f_{m}\right\|_{L_{r}^{2}}\left\|v_{m}\right\|_{L_{r}^{2}},
$$

so that

$$
\left|\left(f_{m}, v_{m}\right)_{N}-\left(f_{m}, v_{m}\right)_{r}\right| \leq C\left[\left\|f_{m}-\Pi_{N-1} f_{m}\right\|_{L_{r}^{2}}+\left\|f_{m}-I_{N} f_{m}\right\|_{L_{r}^{2}}\right]\left\|v_{m}\right\|_{L_{r}^{2}} .
$$

By Theorems 3.3 and 4.4, we conclude that

$$
\sum_{m=-N}^{0}\left|\left(f_{m}, v_{m}\right)_{N}-\left(f_{m}, v_{m}\right)_{r}\right| \leq C N^{-p}\|\mathbf{f}\|_{p}\|\mathbf{v}\|_{0} .
$$

In order to estimate the second sum, we need a lemma.

Lemma 4.6. For any $p \geq 0$, for any $m,-1 \leq m \leq N-2$, for any $\varphi$ in $H_{r}^{p}, R_{m} \varphi$ belongs to $H_{r}^{p}$ and

$$
\left\|R_{m} \varphi\right\|_{H_{r}^{p}} \leq 2\|\varphi\|_{H_{r}^{p}} .
$$


SPECTRAL METHODS IN POLAR COORDINATES FOR THE STOKES PROBLEM 525

Let us assume the lemma. For $1 \leq m \leq N-2, I_{N}\left(R_{m} f_{m}\right)$ belongs to $\mathbf{P}_{N}$ and $D_{m} v_{m}$ belongs to $\mathbf{P}_{N-1}$, so

$$
\left(R_{m} f_{m}, D_{m} v_{m}\right)_{N}-\left(R_{m} f_{m}, D_{m} v_{m}\right)_{r}=\left(I_{N}\left(R_{m} f_{m}\right)-R_{m} f_{m}, D_{m} v_{m}\right)_{r}
$$

and by Theorem 4.4,

$$
\sum_{m=1}^{N-2}\left|\left(R_{m} f_{m}, D_{m} v_{m}\right)_{N}-\left(R_{m} f_{m}, D_{m} v_{m}\right)_{r}\right| \leq C N^{-p}\|\mathbf{f}\|_{p}\|\mathbf{v}\|_{X}
$$

Adding (4.27) and (4.29) gives

$$
\left|L(\mathbf{v})-L_{N}(\mathbf{v})\right| \leq C N^{-p}\|\mathbf{f}\|_{p}\|\mathbf{v}\|_{X}
$$

Plugging (4.25), (4.26) and (4.30) in (4.22), we obtain (4.24).

Proof of Lemma 4.6. It is easy to see by induction that, for any $k \geq 0$,

$$
\left(R_{m} \varphi\right)^{(k)}=-\frac{1}{m+k+1}\left\{k \varphi^{(k-1)}+(m+1) R_{m+k+1} \varphi^{(k)}\right\} .
$$

We now use (4.9) to get an upper bound on $\left\|\left(R_{m} \varphi\right)^{(k)}\right\|_{L_{r}^{2}}$ :

$$
\left\|\left(R_{m} \varphi\right)^{(k)}\right\|_{L_{r}^{2}}^{2} \leq 2\left\{\left\|\varphi^{(k-1)}\right\|_{L_{r}^{2}}^{2}+\frac{1}{4}\left\|\varphi^{(k)}\right\|_{L_{r}^{2}}^{2}\right\} .
$$

Summing for $1 \leq k \leq p$ gives (4.28).

\section{COUPLING SPECTRAL METHOD AND TRANSPARENT BOUNDARY CONDITION}

We consider the Stokes problem in the whole plane:

$$
\left\{\begin{array}{l}
-\Delta \mathbf{u}+\nabla p=\mathbf{f} \quad \text { in } \mathbf{R}^{2} \\
\nabla \cdot \mathbf{u}=0 \text { in } \mathbf{R}^{2}
\end{array}\right.
$$

We shall assume $\mathbf{f}$ to be compactly supported in the disc $D(0, R)$ centered at point 0 and of radius $R$. If $\Omega$ is an unbounded domain, $W^{1}(\Omega)$ is defined by

$$
W^{1}(\Omega)=\left\{v \in \mathcal{D}^{\prime}(\Omega), \frac{\mathbf{v}}{\left(1+r^{2}\right)^{1 / 2}\left(1+\ln \left(1+r^{2}\right)\right)} \in L^{2}(\Omega), \nabla v \in L^{2}(\Omega)\right\},
$$

furnished with the natural inner product and norm

$$
\|\mathbf{v}\|_{W^{1}(\Omega)}^{2}=\left\|\frac{\mathbf{v}}{\left(1+r^{2}\right)^{1 / 2}\left(1+\ln \left(1+r^{2}\right)\right)}\right\|_{0}^{2}+\|\nabla v\|_{0}^{2} .
$$

Note that $\mathbf{R} \subset W^{1}(\Omega)$. A result in [15] asserts that if $\mathbf{f}$ belongs to $\left(L_{0}^{2}\left(\mathbf{R}^{2}\right)\right)^{2}$, this problem has a unique solution $(\mathbf{u}, p)$ in $\left(W^{1}\left(\mathbf{R}^{2}\right) / \mathbf{R}\right)^{2} \times L^{2}\left(\mathbf{R}^{2}\right)$. In order to compute $(\mathbf{u}, p)$, we shall introduce a fictitious boundary, the circle centered at point 0 and of radius $R$, and solve the Stokes problem in $D(0, R)$ with the so-called transparent boundary condition. This boundary condition represents the solution outside the disc. 
5.1. Transparent boundary condition and reduction to a bounded domain. We shall denote $\Omega=D(0, R), \Omega^{\prime}=\mathbf{R}^{2}-\Omega, \Gamma$ their common boundary, $\Gamma=C(0, R)$. The normal vector to $\Gamma$ is oriented toward the exterior of $\Omega$; we shall call it $\mathbf{n}$ (it is $\mathbf{e}_{r}$ with the notations of $\S 2$ ). Problem (5.1) is equivalent to the coupling

$$
\begin{aligned}
& \left\{\begin{array}{l}
-\Delta \mathbf{u}_{1}+\nabla p_{1}=\mathbf{f} \\
\nabla \cdot \mathbf{u}_{1}=0 \quad \text { in } \Omega,
\end{array} \text { in } \Omega,\right. \\
& \left\{\begin{array}{l}
-\Delta \mathbf{u}_{2}+\nabla p_{2}=\mathbf{0} \\
\nabla \cdot \mathbf{u}_{2}=0 \text { in } \Omega^{\prime},
\end{array} \text { in } \Omega^{\prime},\right.
\end{aligned}
$$

with the transmission conditions

$$
\left\{\begin{array}{l}
\mathbf{u}_{1}=\mathbf{u}_{2} \quad \text { on } \Gamma \\
\sigma_{n}\left(\mathbf{u}_{1}\right)=\sigma_{n}\left(\mathbf{u}_{2}\right) \quad \text { on } \Gamma
\end{array}\right.
$$

where $\sigma_{n}$ is the normal strain, i.e.,

$$
\sigma_{n}(\mathbf{u})=\frac{\partial \mathbf{u}}{\partial n}-p \mathbf{n}
$$

Consider the problem

$$
\left\{\begin{array}{l}
-\Delta \mathbf{w}+\nabla q=\mathbf{0} \quad \text { in } \Omega^{\prime} \\
\nabla \cdot \mathbf{w}=0 \text { in } \Omega^{\prime} \\
\mathbf{w}=\mathbf{g} \text { on } \Gamma .
\end{array}\right.
$$

According to [15] again, if $\mathbf{g}$ belongs to $\mathbf{H}^{1 / 2}(\Gamma)$, this problem has a unique solution $(\mathbf{w}, q)$ in $\left(W^{1}\left(\Omega^{\prime}\right)\right)^{2} \times L^{2}\left(\Omega^{\prime}\right)$ and

$$
\|\mathbf{v}\|_{\left(W^{1}\left(\Omega^{\prime}\right)\right)^{2}} \leq C\|\mathbf{g}\|_{H^{1 / 2}(\Gamma)} .
$$

Denote by $K$ the linear operator from $\mathbf{H}^{1 / 2}(\Gamma)$ to $\mathbf{H}^{-1 / 2}(\Gamma)$ defined by $K \mathbf{w}=$ $-\sigma_{n}(\mathbf{w})$. Owing to the transmission conditions on $\Gamma$, problem (5.1) is equivalent to the following boundary value problem in $\Omega$ :

$$
\left\{\begin{array}{l}
-\Delta \mathbf{u}+\nabla p=\mathbf{f} \quad \text { in } \Omega \\
\nabla \cdot \mathbf{u}=0 \quad \text { in } \Omega \\
\sigma_{n}(\mathbf{u})+\mathbf{K u}=0 \quad \text { on } \Gamma
\end{array}\right.
$$

5.2. Basic properties and expression of the transparent operator K. General results valid in any sufficiently smooth geometry assert (see [15] or [9]):

Theorem 5.1. The linear operator $K$ from $\mathbf{H}^{1 / 2}(\Gamma)$ to $\mathbf{H}^{-1 / 2}(\Gamma)$ is continuous, symmetric, positive: for any $\mathbf{g}$ in $\mathbf{H}^{1 / 2}(\Gamma),\langle K \mathbf{g}, \mathbf{g}\rangle_{\Gamma} \geq 0$.

We shall now give the expression of $K$ in polar coordinates. We shall use the notations in $\S 2$, the singularity being here at infinity.

We decompose $\mathbf{g}$ in

$$
\left\{\begin{array}{l}
\mathbf{g}=\mathbf{g}^{(1)}+\mathbf{g}^{(2)} \\
\mathbf{g}^{(1)}(\theta)=\sum_{m \in \mathbf{Z}} g_{m} \mathbf{V}_{m}(\theta), \quad \mathbf{g}^{(2)}(\theta)=\overline{\mathbf{g}}^{(1)}(\theta)=\sum_{m \in \mathbf{Z}} h_{m} \mathbf{W}_{m}(\theta)
\end{array}\right.
$$


SPECTRAL METHODS IN POLAR COORDINATES FOR THE STOKES PROBLEM 527

The function (Euclidean) orthogonal to $\mathbf{g}$ is given by $\mathbf{g}^{\perp}=i\left(-\mathbf{g}^{(1)}+\mathbf{g}^{(2)}\right)$. The Hilbert operator is defined by

$$
\mathcal{H} \mathbf{g}=\sum_{m \in \mathbf{Z}} i \operatorname{sign}(m) g_{m} \mathbf{V}_{m}(\theta)+\sum_{m \in \mathbf{Z}} i \operatorname{sign}(m) h_{m} \mathbf{W}_{m}(\theta)
$$

(with the convention, $\operatorname{sign}(m)=0$ if $m=0$ ).

Theorem 5.2. The operator $K$ is given by

$$
K \mathbf{g}=\frac{1}{R} \frac{\partial}{\partial \theta}\left(\mathbf{g}^{\perp}+\mathcal{H} \mathbf{g}\right)
$$

or in extended form,

$$
\begin{aligned}
K \mathbf{g}=\frac{1}{R}\left[\sum_{m \leq-1}-\right. & m g_{m} \mathbf{V}_{m}(\theta)+\sum_{m \geq 1} 3 m g_{m} \mathbf{V}_{m}(\theta) \\
& \left.+\sum_{m \leq-1}-3 m h_{m} \mathbf{W}_{m}(\theta)+\sum_{m \geq 1} m h_{m} \mathbf{W}_{m}(\theta)\right] .
\end{aligned}
$$

Proof. Since g is given by (5.6), we solve (5.4) in polar coordinates. The first step is to notice that $q$ is harmonic in $\Omega^{\prime}$ and belongs to $L_{r}^{2}(1,+\infty)$. Thus, $q=$ $\sum_{m \in \mathbf{Z}} q_{m} H_{m}$, with

$$
q_{m}=\left\{\begin{array}{l}
\alpha_{m} r^{-|m|}, \quad|m| \geq 2 \\
0, \quad|m| \leq 1
\end{array}\right.
$$

With the notations given in (2.9), the first equation gives

$$
\forall m \in \mathbf{Z}, \quad D_{m} v_{m}-q_{m+1}=c_{m} r^{-(m+1)} .
$$

For any $m \leq 0, r^{-(m+1)}$ does not belong to $L_{r}^{2}(1,+\infty)$; therefore, $c_{m}=0$ :

$$
\forall m \leq 0, \quad D_{m} v_{m}-q_{m+1}=0 .
$$

For any $m \geq 1$, integrate (5.11) from $r$ to $+\infty$ and use (5.10). Then

$$
v_{m}=-\frac{1}{2 m}\left(c_{m}+\alpha_{m+1}\right) r^{-m}, \quad \text { and } \forall m \geq 1, \quad D_{m} v_{m}=-2 m \frac{v_{m}}{r} .
$$

The divergence-free condition reads, $\forall m \in \mathbf{Z}, D_{m-1} v_{m-1}+D_{-m-1} w_{m+1}=0$; thus,

$$
\forall m \geq 1, \quad D_{m} v_{m}=-2 m \frac{v_{m}}{r}=-q_{m+1} .
$$

The operator $K$ is now defined by

$$
\begin{aligned}
K \mathbf{g}= & \sum_{m \in \mathbf{Z}}\left(q_{m+1}-D_{m} v_{m}-m \frac{v_{m}}{r}\right)(R) \mathbf{V}_{m} \\
& +\sum_{m \in \mathbf{Z}}\left(q_{m-1}-D_{-m} w_{m}+m \frac{w_{m}}{r}\right)(R) \mathbf{W}_{m}
\end{aligned}
$$

which gives (5.9). The compact formulation (5.8) comes in a straightforward way from (5.9). 
5.3. Weak formulation of (5.4). For simplicity, we shall assume from now on that $R=1$. We define on $H^{1}(\Omega)$ a bilinear form $\tilde{a}$ by

$$
\tilde{a}(\mathbf{u}, \mathbf{v})=a(\mathbf{u}, \mathbf{v})+\langle K \mathbf{u}, \mathbf{v}\rangle_{\Gamma}
$$

Lemma 5.1. The bilinear form $\tilde{a}$ defines on $\left(H^{1}(\Omega) / \mathbf{R}\right)^{2}$ a scalar product. The corresponding norm is equivalent to the natural norm in $\mathbf{H}^{1}(\Omega)$.

The proof is straightforward, since $K$ is positive, and the $L^{2}$-norm of the gradient is equivalent to the $H^{1}$-norm in $H^{1}(\Omega) / \mathbf{R}$.

The Hilbert space $X$ is $\left(H^{1}(\Omega) / \mathbf{R}\right)^{2}$, furnished with the bilinear form $\tilde{a}, M=$ $\mathbf{L}^{2}(\Omega)$. The variational formulation of problem (5.5) reads:

find $(\mathbf{u}, p)$ in $X \times M$ such that

$$
\begin{cases}\forall \mathbf{v} \in X, & \tilde{a}(\mathbf{u}, \mathbf{v})+b(\mathbf{v}, p)=\langle\mathbf{f}, \mathbf{v}\rangle, \\ \forall q \in M, & b(\mathbf{u}, q)=0 .\end{cases}
$$

Theorem 5.3. Problem (5.13) has a unique solution. In particular, one has the Babuška-Brezzi condition

$$
\inf _{q \in M} \sup _{\mathbf{v} \in X} \frac{b(\mathbf{v}, q)}{\|\mathbf{v}\|_{X}\|q\|_{M}}=1 .
$$

Proof. By Theorem B, it is enough to prove (5.14). We proceed as in (2.5):

$$
\inf _{q \in M} \sup _{\mathbf{v} \in X} \frac{b(\mathbf{v}, q)}{\|\mathbf{v}\|_{X}\|q\|_{M}}=\inf _{q \in M} \frac{\|\mathbf{w}\|_{X}}{\|q\|_{M}}
$$

where $\mathbf{w}$ is the unique solution in $X$ to

$$
\left\{\begin{array}{l}
\mathbf{w} \in H^{1}(\Omega), \\
\Delta \mathbf{w}+\nabla q=\mathbf{0} \quad \text { in } \Omega, \\
\frac{\partial \mathbf{w}}{\partial r}+q \mathbf{e}_{r}+K \mathbf{w}=0 \quad \text { on } \Gamma .
\end{array}\right.
$$

We shall express w in $X$ in terms of $q$ in $M$. Here, $q$ and $\mathbf{w}$ are given by (2.7), (2.8), and the norms are easily computed:

$$
\begin{aligned}
a(\mathbf{w}, \mathbf{w}) & =\sum_{m \in \mathbf{Z}}\left\|D_{m} v_{m}\right\|_{L_{r}^{2}}^{2}+\sum_{m \in \mathbf{Z}} m\left|v_{m}(1)\right|^{2}, \\
\langle K \mathbf{w}, \mathbf{w}\rangle_{\Gamma} & =3 \sum_{m \geq 1} m\left|v_{m}(1)\right|^{2}-\sum_{m \leq-1} m\left|v_{m}(1)\right|^{2}, \\
\tilde{a}(\mathbf{w}, \mathbf{w}) & =\sum_{m \in \mathbf{Z}}\left\|D_{m} v_{m}\right\|_{L_{r}^{2}}^{2}+4 \sum_{m \geq 1} m\left|v_{m}(1)\right|^{2} .
\end{aligned}
$$

The same arguments as in (2.13a) prove

$$
\begin{gathered}
\forall m \in \mathbf{Z}, \quad\left(\frac{d}{d r}+\frac{m+1}{r}\right)\left(q_{m+1}+D_{m} v_{m}\right)=0, \\
\forall m \geq 0, \quad q_{m+1}+D_{m} v_{m}=0 .
\end{gathered}
$$


SPECTRAL METHODS IN POLAR COORDINATES FOR THE STOKES PROBLEM 529

The boundary condition, expanded in the basis $\left\{\mathbf{V}_{m}, \mathbf{W}_{m}\right\}$, gives

$$
\left\{\begin{array}{l}
\text { for } m \leq-1, \quad\left(q_{m+1}+D_{m} v_{m}\right)(1)=0, \\
\text { for } m \geq 0, \quad\left(q_{m+1}+D_{m} v_{m}+4 m v_{m}\right)(1)=0 .
\end{array}\right.
$$

From (5.17), (5.18) and (5.19), we get, for $m \geq 1, v_{m}(1)=0$, and for any $m$ in $\mathbf{Z}$, $\frac{1}{2} q_{m+1}+D_{m} v_{m}=0$. This can be solved explicitly (modulo a constant in $v_{0}$ ):

$$
\begin{gathered}
m \geq 1, \quad v_{m}=\frac{1}{2} r^{m} \int_{r}^{1} \rho^{-m} q_{m+1}(\rho) d \rho, \\
v_{0}=\alpha-\frac{1}{2} \int_{r}^{1} q_{m+1}(\rho) d \rho, \\
m<0, \quad v_{m}=-\frac{1}{2} r^{m} \int_{0}^{r} \rho^{-m} q_{m+1}(\rho) d \rho,
\end{gathered}
$$

and

$$
\|q\|_{0}^{2}=\sum_{m \in \mathbf{Z}}\left\|q_{m}\right\|_{L_{r}^{2}}^{2}=\sum_{m \in \mathbf{Z}}\left\|D_{m} v_{m}\right\|_{L_{r}^{2}}^{2}=a(\mathbf{w}, \mathbf{w}) .
$$

This completes the proof of the theorem.

5.4. The Galerkin method. The discrete spaces are the same as in $\S 3$, i.e.,

$$
\left\{\begin{array}{l}
M_{N}=M \cap\left(\mathbf{S}_{N-1} \otimes \mathbf{P}_{N-1}\right), \\
X_{N}=X \cap H_{N},
\end{array}\right.
$$

and the discrete problem reads: find $\left(\mathbf{u}_{N}, p_{N}\right)$ in $X_{N} \times M_{N}$ such that

$$
\left\{\begin{array}{l}
\forall \mathbf{v} \in X_{N}, a\left(\mathbf{u}_{N}, \mathbf{v}\right)+b\left(\mathbf{v}, p_{N}\right)=L(\mathbf{v}), \\
\forall q \in M_{N}, b\left(\mathbf{u}_{N}, q\right)=0 .
\end{array}\right.
$$

Theorem 5.4. On $X_{N} \times M_{N}$ one has the uniform inf-sup condition

$$
\inf _{q \in M_{N}} \sup _{\mathbf{v} \in X_{N}} \frac{b(\mathbf{v}, q)}{\|\mathbf{v}\|_{X}\|q\|_{M}}=1 .
$$

Proof. We write again

$$
\inf _{q \in M_{N}} \sup _{\mathbf{v} \in X_{N}} \frac{b(\mathbf{v}, q)}{\|\mathbf{v}\|_{X}\|q\|_{M}}=\inf _{q \in M_{N}} \frac{\|\mathbf{w}\|_{X}}{\|q\|_{M}},
$$

where $\mathbf{w}$ is the unique solution to

$$
\forall \mathbf{v} \in X_{N}, \quad-(\Delta \mathbf{w}+\nabla q, \mathbf{v})_{0}+\left\langle K \mathbf{w}+\frac{\partial \mathbf{w}}{\partial r}+q \mathbf{e}_{r}, v\right\rangle_{\Gamma}=0 .
$$

Using formula (5.20a), we can easily see that if $q$ belongs to $M_{N}$, then $\mathbf{w}=$ $-(\Delta)^{-1} \nabla q$ belongs to $X_{N}$. The constant is thus greater than or equal to 1 . The choice $q_{0}=0, q_{m}=r^{m}, 1 \leq m \leq N-1$, gives equality.

Theorem B gives the conclusion:

Theorem 5.5. For any $\mathbf{f}$ in $H^{-1}(\Omega)$, the problem (5.21) has a unique solution $\left(\mathbf{u}_{N}, p_{N}\right)$ in $X_{N} \times M_{N}$ and

$$
\left\|\mathbf{u}_{N}\right\|_{X}+\left\|p_{N}\right\|_{0} \leq C\|\mathbf{f}\|_{-1} .
$$

Slight modifications to the proofs in $\S 3$ give the optimal error estimates: 
Theorem 5.6. For any integer $p \geq 0$, if $\mathbf{f}$ belongs to $\mathbf{H}^{p}(\Omega)$, the solutions $(\mathbf{u}, p)$ and $\left(\mathbf{u}_{N}, p_{N}\right)$ to (5.13) and (5.22) satisfy the optimal error estimates

$$
\begin{gathered}
\left\|\mathbf{u}-\mathbf{u}_{N}\right\|_{X}+\left\|p-p_{N}\right\|_{0} \leq C N^{-1-p}\|\mathbf{f}\|_{p}, \\
\left\|\mathbf{u}-\mathbf{u}_{N}\right\|_{0} \leq C N^{-2-p}\|\mathbf{f}\|_{p} .
\end{gathered}
$$

Remark 5.1. By scaling we can solve the problem in $D(0, R)$ with a Galerkin method. This leads to the following error estimates:

$$
\begin{gathered}
\left\|\mathbf{u}-\mathbf{u}_{N}\right\|_{X}+\left\|p-p_{N}\right\|_{0} \leq C\left(\frac{N}{R}\right)^{-1-p}\|\mathbf{f}\|_{p}, \\
\left\|\mathbf{u}-\mathbf{u}_{N}\right\|_{0} \leq C\left(\frac{N}{R}\right)^{-2-p}\|\mathbf{f}\|_{p} .
\end{gathered}
$$

\section{Conclusion}

This is a first step toward the solution of exterior problems by spectral methods in a bounded domain. The second step should be to deal with operators with nonconstant coefficients, and the third step the three-dimensional case, with the use of spherical harmonic functions. This will be of great use, for instance in meteorology.

\section{REFERENCES}

1. C. Bernardi and Y. Maday, Approximations spectrales de problèmes aux limites elliptiques, Mathématiques et Applications, vol. 10, Springer-Verlag, Paris, 1992. MR 94f:65112

2. _ A collocation method over staggered grids for the Stokes problem, Internat. J. Numer. Methods Fluids 8 (1988), 537-557. MR 89j:76041

3. H. Brezis, Analyse fonctionnelle: théorie et applications, Masson, Paris, 1983. MR 85a:58022

4. C. Canuto, S. I. Hariharan and L. Lustman, Spectral methods for exterior elliptic problems, Numer. Math. 46 (1985), 505-520. MR 86j:65148

5. C. Canuto and A. Quarteroni, Approximation results for orthogonal polynomials in Sobolev spaces, Math. Comp. 38 (1982), 67-86. MR 82m:41003

6. P. J. Davis and P. Rabinowitz, Methods of numerical integration, 2nd ed., Academic Press, Orlando, 1984. MR 86d:65004

7. J. Dieudonné, Calcul infinitésimal, Hermann, Paris, 1968. MR 37:2557

8. V. Girault and P.-A. Raviart, Finite element methods for Navier-Stokes equations: theory and algorithms, Springer Series in Comput. Math., vol. 5, Springer-Verlag, Berlin and New York, 1986. MR 88b:65129

9. L. Halpern, Méthodes spectrales pour la résolution du problème de Stokes dans un disque, Application aux calculs en domaine non borné, Prépublication 93-12, Université Paris-Nord.

10. C. Johnson and J. C. Nedelec, On the coupling of boundary integral and finite element methods, Math. Comp. 35 (1980), 1063-1079. MR 82c:65072

11. J. B. Keller and D. Givoli, Exact nonreflecting boundary conditions, J. Comput. Phys. 82 (1989), 172-192. MR 91a:76064

12. M. Lenoir and A. Tounsi, The localized finite element method and its application to the twodimensional sea-keeping problem, SIAM J. Numer. Anal. 25 (1988), 729-752. MR 89h:90046

13. V. Levillain, Couplage éléments finis-équations intégrales pour la résolution des équations de Maxwell en milieu hétérogène, Thèse de doctorat de l'Ecole Polytechnique, June 1991.

14. B. Mercier and G. Raugel, Résolution d'un problème aux limites dans un ouvert axisymétrique par éléments finis en $r, z$ et séries de Fourier en $\theta$, RAIRO Anal. Numér. 16 (1982), 405-461. MR 84g:65154

15. A. Sequeira, The coupling of boundary integral and finite element methods for the bidimensional exterior steady Stokes problem, Math. Methods Appl. Sci. 5 (1983), 356-376. MR 85g:65121 
SPECTRAL METHODS IN POLAR COORDINATES FOR THE STOKES PROBLEM 531

16. G. Szegö, Orthogonal polynomials, Amer. Math. Soc. Colloq. Publ., vol. 23, Amer. Math. Soc., Providence, RI, 1939. MR1:14

17. H. Vandeven, Compatibilité des espaces discrets pour l'approximation spectrale du problème de Stokes périodique/non périodique, RAIRO Modél. Math. Anal. Numer. 23 (1989), 649-688. MR 91h:65185

Université Paris-Nord, Institut Galilée, Département de Mathématiques, Laboratoire "Analyse, Géométrie et Applications", URA 742 du CNRS, 93430 Villetaneuse, FRANCE

E-mail address: halpern@math.univ-paris13.fr 